Article

\title{
Cu-Doped KCl Unfolded Band Structure and Optical Properties Studied by DFT Calculations
}

\author{
César Castillo-Quevedo ${ }^{1}$, Jose Luis Cabellos ${ }^{2, *}$, Raul Aceves ${ }^{2}$, Roberto Núñez-González ${ }^{3}$ and \\ Alvaro Posada-Amarillas $2, *$ (D) \\ 1 Departamento de Fundamentos del Conocimiento, Centro Universitario del Norte, Universidad de \\ Guadalajara, Carretera Federal No. 23, Km. 191, C.P. 46200 Colotlán, Jalisco, Mexico; \\ castillo.quevedo@cunorte.udg.mx \\ 2 Departamento de Investigación en Física, Universidad de Sonora, Blvd. Luis Encinas y Rosales S/N, \\ 83000 Hermosillo, Sonora, Mexico; raul.aceves@unison.mx \\ 3 Departamento de Matemáticas, Universidad de Sonora, Blvd. Luis Encinas y Rosales S/N, 83000 Hermosillo, \\ Sonora, Mexico; ronunez@mat.uson.mx \\ * Correspondence: jose.cabellos@unison.mx (J.L.C.); posada@cifus.uson.mx (A.P.-A.)
}

Received: 17 July 2020; Accepted: 22 September 2020; Published: 26 September 2020

\begin{abstract}
The unfolded band structure and optical properties of $\mathrm{Cu}$-doped $\mathrm{KCl}$ crystals were computed by first principles within the framework of density functional theory, implemented in the ABINIT software program, utilizing pseudopotential approximation and a plane-wave basis set. From a theoretical point of view, $\mathrm{Cu}$ substitution into pristine $\mathrm{KCl}$ crystals requires calculation by the supercell (SC) method. This procedure shrinks the Brillouin zone, resulting in a folded band structure that is difficult to interpret. To solve this problem and gain insight into the effect of copper ions $\left(\mathrm{Cu}^{+}\right)$on electronic properties, the band structure of SC KCl:Cu was unfolded to make a direct comparison with the band structure of the primitive cell (PC) of pristine $\mathrm{KCl}$. To understand the effect of $\mathrm{Cu}$ substitution on optical absorption, we calculated the imaginary part of the dielectric function of $\mathrm{KCl}: \mathrm{Cu}$ through a sum-over-states formalism and broke it down into different band contributions by partially making an iterated cumulative sum (ICS) of selected valence and conduction bands. Consequently, we identified those interband transitions that give rise to the absorption peaks due to the $\mathrm{Cu}^{+}$ion. These transitions involve valence and conduction bands formed by the $\mathrm{Cu}-3 \mathrm{~d}$ and $\mathrm{Cu}-4 \mathrm{~s}$ electronic states.
\end{abstract}

Keywords: DFT calculations; unfolding band structure; optical spectrum; $\mathrm{KCl}$

\section{Introduction}

Alkali halide (AH) crystals are solids of great importance from theoretical and experimental points of view. They are of great research interest in solid-state physics [1], mainly due to their high stability [2]. Pure AH crystals are relatively easy to produce in large quantities. They possess high melting points, varying from 600 to $1000{ }^{\circ} \mathrm{C}$ [3], are poor conductors of heat [2], and have strong miscibility in polar media [4]. They are also the most ionic of all crystal compounds [2] that consist of ions bound together by electrostatic attraction, making them good candidates for studying other systems [5]. The AH crystals have a large energy gap in the order of 8-10 eV, making them useful for the development of laser optical components as optical transmission windows in the ultraviolet (UV) to infrared (IR) ranges of the electromagnetic spectrum [3,6], among other optical applications. AH crystals, either pure or doped, are also employed as neutron monochromators [3].

Potassium chloride $(\mathrm{KCl})$ is an inorganic salt with properties similar to those of common salt $(\mathrm{NaCl})$ [7]. $\mathrm{KCl}$ is employed in optical windows for laser applications [8], and as a scintillator 
or in dosimetry by adding impurities [9-11]. At the end of the 1960s, several experimental and theoretical studies on the optical properties of $\mathrm{KCl}$ and other $\mathrm{AH}$ crystals were presented [12-19]. Recently, theoretical predictions on novel $\mathrm{KCl}$ phases under pressure have been proposed [20]. Several studies have addressed doping of $\mathrm{KCl}$ with monovalent cations, e.g., $\mathrm{Ga}^{+}$, $\mathrm{In}^{+}$, and $\mathrm{Tl}^{+}$, to investigate distortion trends as a function of impurity [21]. Furthermore, recently published works show an enhancement in the optical properties of $\mathrm{KCl}$ crystals when doped with $\mathrm{ZnO}$ [11]. In other previous works, the optical properties of $\mathrm{KCl}$ crystals doped with $\mathrm{Sb}_{2} \mathrm{O}_{3}$ nanocrystals were investigated, analyzing the doping effects of the $\mathrm{Sb}_{2} \mathrm{O}_{3}$ nano-semiconductor on the optical properties of $\mathrm{KCl}$ crystals [22]. The optical properties of copper ion $\left(\mathrm{Cu}^{+}\right)$-doped $\mathrm{KCl}$ and other $\mathrm{AH}$ crystals have also been studied due to their outstanding UV-light absorption properties [23-25], a characteristic that makes them excellent phosphor materials for many technological applications [26-28]. In the $\mathrm{Cu}$-doped $\mathrm{KCl}$, several absorption bands in the UV region have been observed, with a strong dependence on temperature $[23,29]$. These bands have been attributed to $3 d^{10} \rightarrow 3 d^{9} 4 s^{1}$ transition states of $\mathrm{Cu}^{+}$. Theoretical calculations of the oscillator strength of $\mathrm{Cu}^{+} 3 \mathrm{~d}^{10} \rightarrow 3 \mathrm{~d}^{9} 4 \mathrm{~s}^{1}$ transitions in $\mathrm{NaF}$ hosts were performed to verify this conclusion [30]. Recently, density functional theory (DFT) calculation of the electronic and optical properties of $\mathrm{LiF}: \mathrm{Cu}^{+}$was carried out, employing the materials modelling code based on a quantum mechanical description of electrons (CASTEP code) within the pseudopotential approximation and reporting the formation of a band in the middle of the electronic bandgap due to copper $(\mathrm{Cu})$ [31]. Despite the remarkable efforts that have been made to elucidate the electronic structure and origin of the absorption and emission spectra of the $\mathrm{Cu}^{+}$ion embedded in $\mathrm{KCl}$ and other AH crystals, this has still not been achieved, and further theoretical studies with different models and methodologies are needed.

In this paper, we report the results of DFT calculations of the band structure, density of states, and optical properties of the $\mathrm{Cu}^{+}$ion embedded in $\mathrm{KCl}$. To study and identify the effects that impurities have on its electronic structure, we unfolded the band structure of $\mathrm{KCl}: \mathrm{Cu}$ and compared it with that of pristine $\mathrm{KCl}$. The band-to-band contribution to the optical spectrum is examined by partially summing selected bands using the sum-over-states formalism [32], which is employed in the evaluation of the imaginary part of the linear electric susceptibility tensor. The transitions between valence and conduction bands that are responsible for the effects of $\mathrm{Cu}$ substitution on the absorption of $\mathrm{KCl}$ are thus identified. The remainder of this paper is organized as follows: Section 2 briefly outlines the theory and provides the computational details. Section 3 presents a detailed discussion of the results of the unfolding and folding band structure, density of states, and band-to-band contribution to the optical response of pristine $\mathrm{KCl}$ and doped $\mathrm{KCl}: \mathrm{Cu}$ system. Conclusions are given in Section 4 .

\section{Theoretical Methods and Computational Details}

$\mathrm{KCl}$ is an ionic crystal with a rock salt structure $(\mathrm{NaCl})$. The face-centered cubic (FCC) crystalline structure phase of $\mathrm{KCl}$ belongs to the space group (Hermann-Mauguin) Fm3m (number 225) and point group (Hermann-Mauguin) $\mathrm{m} 3 \mathrm{~m}$. In this structure, an octahedron of six counterions surrounds each ion; hence, the coordination number is six for both the anion, $\mathrm{Cl}^{-}$, and cation, $\mathrm{K}^{+}$. The experimental lattice constant is $6.29 \AA$ [3,33-35]. Figure 1a shows the FCC primitive cell (PC) of pristine $\mathrm{KCl}$, with two atoms at the base; the cation $\mathrm{K}^{+}$is located at the $[0,0,0]$ position, and the anion $\mathrm{Cl}^{-}$is located at the $[1 / 2,1 / 2,1 / 2]$ position, for which the Brillouin zone (BZ) is a truncated octahedron [36,37], and its primitive vectors are $[0,1 / 2,1 / 2],[1 / 2,0,1 / 2]$, and $[1 / 2,1 / 2,0]$. Figure $1 b$ shows a larger FCC pristine system that contains eight times as many atoms as in the PC, so the number of atoms is $16 \mathrm{C}^{\mathrm{Cl}^{-}}$and $8 \mathrm{~K}^{+}$). This cubic phase was obtained by accurately multiplying the PC lattice constants of $\mathrm{KCl}$ by $2 \times 2 \times 2$. As a result, the lattice vector length became twice that of the original unit cell of the PC and had the same primitive vectors. This system allows us to make $\mathrm{Cu}$ substitutions, doping the $\mathrm{KCl}$ structure. Figure $1 \mathrm{c}$ shows an FCC doped system that contains eight anions of $\mathrm{Cl}^{-}$, seven cations of $\mathrm{K}^{+}$, and one monovalent cation of $\mathrm{Cu}^{+}$, occupying the $[0,0,0]$ position of the substituted cation $\mathrm{K}^{+}$. 


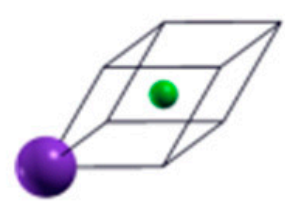

(a)

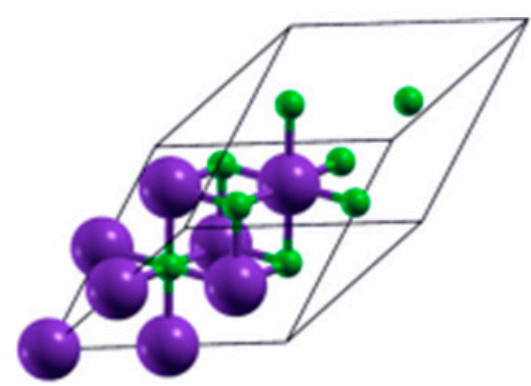

(b)

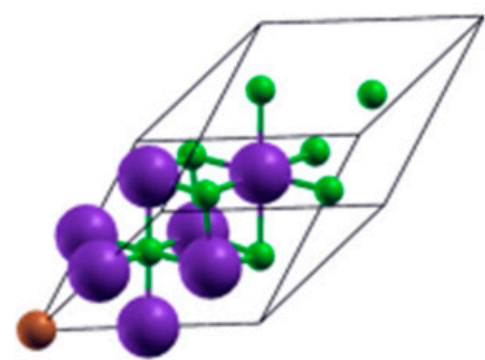

(c)

Figure 1. (a) Pristine face-centered cubic (FCC) primitive cell of $\mathrm{KCl}$ with two atoms at the base, (b) $2 \times 2 \times 2$ pristine FCC supercell of the $\mathrm{KCl}$ with 16 atoms at the base, and (c) FCC supercell of $\mathrm{KCl}$ doped with $\mathrm{Cu}^{+}$atom with 16 atoms at the base. The violet-, green-, and copper-colored spheres represent the potassium, chlorine, and copper atoms, respectively. The unit cell size are depicted proportionally.

To gain insight into the effect of $\mathrm{Cu}$ dopant in electronic and optical properties of pure $\mathrm{KCl}$, we consider the exploration of the other seven possible sites that the $\mathrm{Cu}$ atom replaces the host cation $\mathrm{K}$ shown in Figure S1. We computed the geometric, electronic, and optical properties for those eight systems. The doping position affects the bandgap, and optical properties of the $\mathrm{KCl}: \mathrm{Cu}$ system. Interesting, it induces an anisotropic nanostructure, as a consequence, induces optical anisotropy.

In the following, we studied the doped isotropic system of Figure 1c, and the doped anisotropic system shows in Figure S1b of the supplementary material. With these doped systems, we calculate the optical and electronic properties, and the electronic and optical properties of the pristine supercell SC/PC work as a reference system to observe the effect of the $\mathrm{Cu}^{+}$ion. Particular attention should be paid to the SC method of calculation. This method conveniently allows the folding of the bands into the smaller SC BZ that gives rise to a complicated band structure for which it is difficult to determine whether the bandgap is direct or indirect $[38,39]$. Moreover, other physical quantities, such as carrier mobility [40], require knowledge of the PC band structure. Several authors have already recently developed theories and methods with diverse approximations to unfold the SC band structure into a PC band structure, with successful results [39,41-53]. In this study, the unfolding of the SC BZ into the PC BZ was performed using the BandUP computer program $[39,49,50]$, which is based on the evaluation of the spectral weight,

$$
P_{m K}(\vec{k})=\sum_{n}\left|\left\langle\psi_{m k}^{P C} \mid \psi_{n K}^{S C}\right\rangle\right|^{2}
$$

where $\left\langle\psi_{m k}^{P C}\right.$ and $\left.\psi_{n K}^{S C}\right\rangle$ are the eigenstates of the PC and SC, respectively and $P_{m K}(\vec{k})$ is the spectral weight, and $\vec{k}$ is a reciprocal k-point. This method gives the probability that an eigenstate of a Hamiltonian, in the SC representation, will have the same character as a PC state. This unfolding procedure of the SC BZ has been successfully employed in several systems, such as SiGe nanowires [38], graphene [54,55], and ternary alloys [56].

The optical absorption spectrum is determined by the dielectric function, $\varepsilon(\omega)$ [57], which is related to the electric susceptibility $\left[\chi_{1}^{a b}(-\omega ; \omega)\right][58]$ by Equation (2)

$$
\varepsilon(\omega)=1+4 \pi\left[\chi_{1}^{a b}(-\omega ; \omega)\right]
$$


where $\mathrm{ab}$ letters are the axes $\mathrm{x}, \mathrm{y}$ and $\mathrm{z}$ of an orthogonal coordinate system and $\omega$ is the frequency of the light. The imaginary part of the electric susceptibility is given by:

$$
\left[\chi_{1}^{a b}(-\omega ; \omega)\right]=\frac{e^{2}}{h} \int \frac{d^{3} k}{8 \pi} \sum_{n \neq m} f_{n m} r_{n m}^{a}(k) r_{m n}^{b}(k) \delta\left(\omega_{m n}-\omega\right)
$$

In Equation (3), $e$ and $h$ are the elementary charge and Planck's constant, respectively; and $n, m$ are the occupied initial and unoccupied final states:

$$
f_{m n}=f_{m}-f_{n}
$$

with $f_{i}$ as the Fermi occupation factor given in Equation (4), which is zero or unity for cold semiconductors;

$$
\omega_{m n}(k)=\omega_{m}(k)-\omega_{n}(k)
$$

are the frequency differences given in Equation (5), where $\omega_{m}(k)$ is the DFT energy of band $m$ at wave vector $k$; and $r_{n m}^{a}(k)$ are the matrix position elements of bands $m$ and $n$ at vector $k$. Following the procedure of a previous work [32], in this study, we computed the expression given in Equation (3). The peaks, presented in the $\left[\chi_{1}^{a b}(-\omega ; \omega)\right]$ part, are due to the direct interband optical transitions between the valence and conduction bands, which can be identified from the critical points of the band structure. Moreover, in this study, the bands that can contribute significantly to the $\left[\chi_{1}^{a b}(-\omega ; \omega)\right]$ part are identified by breaking down the $\left[\chi_{1}^{a b}(-\omega ; \omega)\right]$ part into different band contributions $[59,60]$. In addition, we considered the scissor correction [32,61], the approximation of which is obtained by a rigid shift of the DFT energies to an experimental value [62]. Therefore, the spectrum of $\left[\chi_{1}^{a b}(-\omega ; \omega)\right]$ is rigidly shifted along the energy axis without changing the spectrum shape [32,61]. The real part $\left[\chi_{1}^{a b}(-\omega ; \omega)\right]$ was computed using Kramers-Kronig relations [63]. Here, the indirect transitions are neglected because they have little contribution [64] to $\left[\chi_{1}^{a b}(-\omega ; \omega)\right]$, and the spin-orbit, local field, and electron-hole effects are also neglected $[32,65]$. The inclusion of these effects is beyond the scope of this study.

The electronic and optical properties are calculated using the SC approach in the DFT framework, as is implemented in the freely available ABINIT software program $[66,67]$. We chose the optimized norm-conserving Vanderbilt (ONCV) pseudopotentials [68] taken from the ONCVPSP-PBE-PD v0.4 library, following reference [66]. These were validated against all-electron calculations and found to perform well. Among these pseudopotentials, the $3 s^{2} 3 p^{6} 3 d^{10} 4 s^{1}, 3 s^{2} 3 p^{6} 4 s^{1}$, and $3 s^{2} 3 p^{5}$ electrons of the $\mathrm{Cu}, \mathrm{K}$, and $\mathrm{Cl}$ atoms, respectively, are treated as valence states. We employed the Perdew-Burke-Ernzerhof (PBE) general gradient approximation functional to calculate the exchange-correlation energy [69].

The wavefunctions were expanded in a plane-wave basis set and checked for convergence by applying a kinetic cutoff energy of $25 \mathrm{Ha}$. The Monkhorst-Pack scheme [70] was used to sample the irreducible Brillouin zone (IBZ). The IBZ integration was performed by employing the tetrahedron method [32]. Table 1 shows the number of the chosen $k$-points, cutoff energy, and $k$-point mesh corresponding to the system, to assure convergence of the total energy and forces as well as the optical properties. The total energy of the self-consistent field (SCF) procedure was set to a value of $5 \times 10^{-7} \mathrm{eV} /$ atom. All the atomic positions of the studied systems were relaxed until the Hellmann-Feynman forces on each atom were lower than $20 \mathrm{meV} / \AA$. The resulting atomic structures were used for all the calculations presented in this study. 
Table 1. Parameters employed in the present study's calculation.

\begin{tabular}{cccc}
\hline Unit Cell. & Number of $\boldsymbol{k}$-Points & Size Grid & Energy Cutoff (Ha) \\
\hline (a) $\mathrm{KCl}$ & 3864 & $55 \times 55 \times 55$ & 25 \\
(b) $\mathrm{K}_{8} \mathrm{Cl}_{8}$ & 2920 & $19 \times 19 \times 19$ & 25 \\
(c) $\mathrm{K}_{7} \mathrm{Cl}_{8}: \mathrm{Cu}$ & 2920 & $19 \times 19 \times 19$ & 25 \\
\hline
\end{tabular}

\section{Results and Discussion}

\subsection{Optimized Lattice Constant}

We computed the PC KCl lattice constant through minimization of the total energy by employing the parameters shown in Table 1. The obtained result was $4.507 \AA$ for the FCC PC system, which is in agreement with the experimental lattice constant of $6.29 \AA$ [3,33-35] - larger by $1.22 \%$ and is slightly shorter by $0.3 \%$-compared with other theoretical calculations that obtained $4.522 \AA$ [71]. It is more similar to other calculations that used an all-electron scheme [28] (see Table 2). The relation between cells FCC-PC and cubic SC with eight atoms basis is a factor of 1.4142. $(4.507 \times 1.4142=6.37)$. Figure S2 shows the pure $\mathrm{KClSC}$ on the basis of eight atoms.

Table 2. Lattice constant and bandgap obtained in the DFT calculation for all systems where Cu dopant occupies substitutions sites replacing host $\mathrm{K}$ cations.

\begin{tabular}{cccc}
\hline Figure. & Unit Cell & Lattice Constant (Å) & Bandgap (eV) \\
\hline Figure 1a & $\mathrm{KCl}$ & 4.507 & 5.07 \\
Figure 1b & $\mathrm{K}_{8} \mathrm{Cl}_{8}$ & 9.014 & 5.07 \\
Figure 1c & $\mathrm{K}_{7} \mathrm{Cl}_{8}: \mathrm{Cu}$ & 8.390 & 2.20 \\
Figure S1b & $\mathrm{K}_{7} \mathrm{Cl}_{8}: \mathrm{Cu}$ & 8.480 & 3.20 \\
Figure S1c & $\mathrm{K}_{7} \mathrm{Cl}_{8}: \mathrm{Cu}$ & 8.480 & 3.20 \\
Figure S1d & $\mathrm{K}_{7} \mathrm{Cl}_{8}: \mathrm{Cu}$ & 8.580 & 3.20 \\
Figure S1e & $\mathrm{K}_{7} \mathrm{Cl}_{8}: \mathrm{Cu}$ & 8.480 & 3.20 \\
Figure S1f & $\mathrm{K}_{7} \mathrm{Cl}_{8}: \mathrm{Cu}$ & 8.480 & 3.20 \\
Figure S1g & $\mathrm{K}_{7} \mathrm{Cl}_{8}: \mathrm{Cu}$ & 8.480 & 3.20 \\
Figure S1h & $\mathrm{K}_{7} \mathrm{Cl}_{8}: \mathrm{Cu}$ & 8.394 & 2.20 \\
\hline
\end{tabular}

The FCC supercell of the pristine system $\mathrm{K}_{8} \mathrm{Cl}_{8}$ is eight times the volume of the primitive cell of $\mathrm{KCl}$. In Table 2, the lattice constant of the SC two times greater than the lattice constant of the PC. After the optimization of the $\mathrm{K}_{7} \mathrm{Cl}_{8}: \mathrm{Cu}$ system, the resulting unit cell is FCC with its three optimized lattice parameters measuring $8.390 \AA$, and its three lattice angles being $60^{\circ}$. The optimized volume of the pristine $\mathrm{K}_{8} \mathrm{Cl}_{8}$ is $472.0 \AA^{3}$, and the volume of $\mathrm{K}_{7} \mathrm{Cl}_{8}: \mathrm{Cu}$ is $417.7 \AA^{3}$. The SC volume of the doped system is $11.5 \%$ smaller, which is evidently due to copper doping (in Figure 1, this can be seen in the proportions of the unit cells). For this system, the calculated density of $\mathrm{Cu}$ based on its atomic weight and the volume of the unit cell with an $8.390 \AA$ edge length is $0.25 \mathrm{~g} / \mathrm{cm}^{3}$. The unit cell has five non-equivalent atoms, and the distances between the $\mathrm{Cu}$ atom and the ions surrounding it are shorter by approximately $0.53 \AA$. The optimized bond length of $\mathrm{K}-\mathrm{Cu}$ is $2.651 \AA$ compared to the bond length of $\mathrm{K}-\mathrm{Cl}$ atoms ( $3.187 \AA$ ) in the pristine system. In Tables S1-S3 is given the list of non-equivalent atoms in the unit cell, the list of atoms in the unit cell and the three lattice vectors, respectively, of the $\mathrm{KCl}: \mathrm{Cu}$ system of Figure 1c. The exploration of the other possible sites after optimization indicate that there are two optimized lattice parameters shown in Table 2 and that there is a relative displacement of doped and host atoms as a function of dopant occupying the site. The displacement at each lattice site depends on the $\mathrm{Cu}$ atom occupying that site and the which kind of atoms are closer to the $\mathrm{Cu}$ atom. To better appreciate, Figure S3 shows the coordination polyhedron for each system. It is clear that, if we take the polyhedron of the pure $\mathrm{KCl}$ as a reference, the eight polyhedra of the $\mathrm{Cu}$ doped systems are distorted. On a closer inspection of Figure S3, we can distinguish that there are two shapes of coordinate polyhedra. Figure $\mathrm{S}^{3} \mathrm{a}_{1}$ shows regular spherical polyhedron, where the optimized bond 
length between the $\mathrm{Cu}$ atom and the six surrounding $\mathrm{Cl}$ atoms is $2.651 \AA$. Figure $\mathrm{S} 3 \mathrm{~b}_{1}$ shows a prolate polyhedron, where there are two types of optimized bond length among the $\mathrm{Cu}$, and six surrounding $\mathrm{Cl}$ atoms, four optimized bound lengths of $\mathrm{Cu}-\mathrm{Cl}$ are $3.146 \AA$; the other two are $2.151 \AA$. The rest of the polyhedra are of one type or another. The $\mathrm{Cu}$ dopant occupying different sites of the $\mathrm{KCl}$ gives rise to two different geometrical systems that cause significant changes in their electronic and optical properties of the $\mathrm{KCl}$.

\subsection{Electronic Band Structure and Density of States}

The band structure of the pristine $\mathrm{KCl}$ has been reported previously $[28,35,71]$. For comparison, in later sections with the doped system, we show in Figure 2a the band structure (blue lines) of the $\mathrm{PC}$ for $\mathrm{KCl}$ computed along high-symmetry directions in the $\mathrm{BZ}$, from the $\mathrm{BZ}$ center $\Gamma$ with the coordinates $(0,0,0)$ to the $X$ point $(0,0.5,0.5), \mathrm{W}$ point $(0.25,0.5,0.75), \mathrm{K}$ point $(0.375,0.375,0.75)$, $\Gamma$ point $(0.0,0.0,0.0), \mathrm{L}$ point $(0.0,0.0,0.5), \mathrm{U}$ point $(0.0,0.375,0.625), \mathrm{W}$ point $(0.25,0.5,0.75)$, $\mathrm{L}$ point $(0.0,0.0,0.5), \mathrm{U}$ point $(0.0,0.375,0.625)$, and $X$ point $(0.00 .50 .5)$ in units of $2 \pi / a, 2 \pi / b, 2 \pi / c$, where $a, b$, and $c$ are the lattice constants shown in Table 2. Figure 2 a shows the unfolded band structure of the pristine $\mathrm{SC} \mathrm{K}_{8} \mathrm{Cl}_{8}$ (black lines) superimposed to the band structure of pristine PC. Figure $2 \mathrm{~b}$ shows a zoomed view in the region -1.5 to $0.75 \mathrm{eV}$. The lowest direct Kohn-Sham band gap of the $\mathrm{KCl}$ obtained in both calculations is $\sim 5.07 \mathrm{eV}$, at the $\Gamma$ point, as indicated by the red arrow in Figure 2a (displayed in Table 2) and in agreement with previous calculations [35,71].

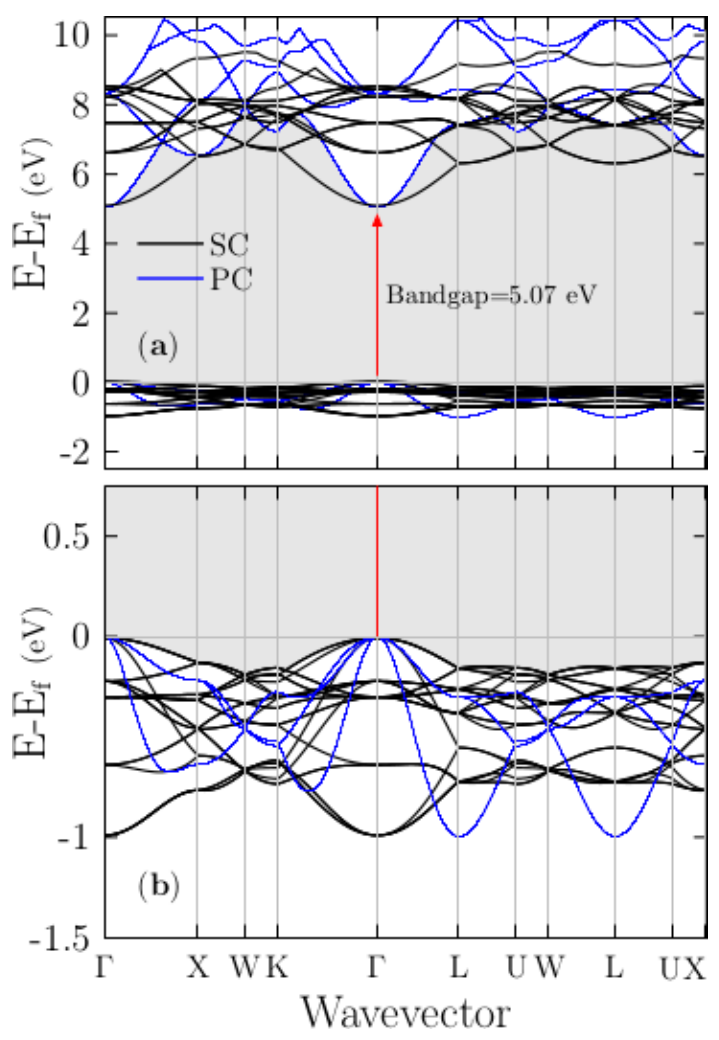

Figure 2. The upper panel (a) shows the band structure of the primitive cell (PC) for $\mathrm{KCl}$ (blue lines) and the folded band structure of the supercell (SC) of pristine $\mathrm{K}_{8} \mathrm{Cl}_{8}$ (black lines) along with the same high-symmetry directions of the Brillouin zone (BZ) of the PC. The lower panel (b) shows a zoomed view in the region -1.5 to $0.75 \mathrm{eV}$. The shortest direct Kohn-Sham bandgap indicated by the red arrow is $5.07 \mathrm{eV}$ at the $\Gamma$ point. The blue lines correspond to the PC band structure, and the black lines correspond to the SC band structure. Figure S4 of the supplementary information, we show the band structure with applied scissors correction. 
In these calculations, the bandgap is underestimated by $\sim 3.53 \mathrm{eV}$ compared with the experimental value of $8.6 \mathrm{eV}[72,73]$ of the $\mathrm{KCl}$, and other recently reported all-electron DFT calculations using the modified Becke-Johnson approximation [28]. To correct the bandgap that was underestimated by DFT [74], we applied a scissor correction of $3.53 \mathrm{eV}$ to the conduction bands, which shifted them to the experimental value. Following and comparing the results presented in Figure $2 a, b$, it can be seen that the principal difference is the band dispersion in the $\mathrm{K}-\Gamma-\mathrm{L}$ direction, although we consider the same crystal. This difference is due to SC shrink of the BZ; thus, one should not wrongly conclude something that depends on the dispersion of the band structure (e.g., the effective mass) using an SC band structure.

The inset in the lower Figure $3 \mathrm{~b}$ shows a zoomed view in the range 4.5 to $6.5 \mathrm{eV}$ energy axes, clearly shows that density of states start to raise at energy range of $5 \mathrm{eV}$, as we expected. Notice the main difference: the density is higher in the SC than in the PC system. The color code is violet for K and green for $\mathrm{Cl}$. Figure S5 of the supplementary information, shows the projected density of states (PDOS) with applied scissors correction. To elucidate the nature of the electronic band structure, we calculated the PDOS as a function of energy for the pristine $\mathrm{PC} \mathrm{KCl}$ (Figure 3a), $\mathrm{SC} \mathrm{K}_{8} \mathrm{Cl}_{8}$ (Figure 3b), and doped $\mathrm{SC} \mathrm{K}_{7} \mathrm{Cl}_{8}: \mathrm{Cu}$ (Figure $4 \mathrm{a}-\mathrm{c}$ ) structures. In those plots, the Fermi energy is shifted to zero. For the pristine PC and SC, our results are in good agreement with those of previous studies [28]; notice that the density of PDOS for the SC is higher than that for the PC. The examination of the PDOS shows that the width of valence bands is approximately $1 \mathrm{eV}$, which is in agreement with the folded band structure (black lines) shown in Figure $2 b$. The highest valence bands are mainly formed by the $\mathrm{Cl}-3 \mathrm{p}$ states, which are in line with previous studies [35]. Just slightly below the Fermi level, there are tiny contributions of K-3p states, as can be seen from the PDOS plot in Figure 3. Thus, there is a slight admixture (not an overlap) between the Cl-3p states and a few K-3p states, as expected for an ionic compound. Meanwhile, at approximately $5 \mathrm{eV}$, the bottom conduction bands have contributions from both the $\mathrm{K}-4 \mathrm{~s}$ and $\mathrm{Cl}-4 \mathrm{~s}$ states.

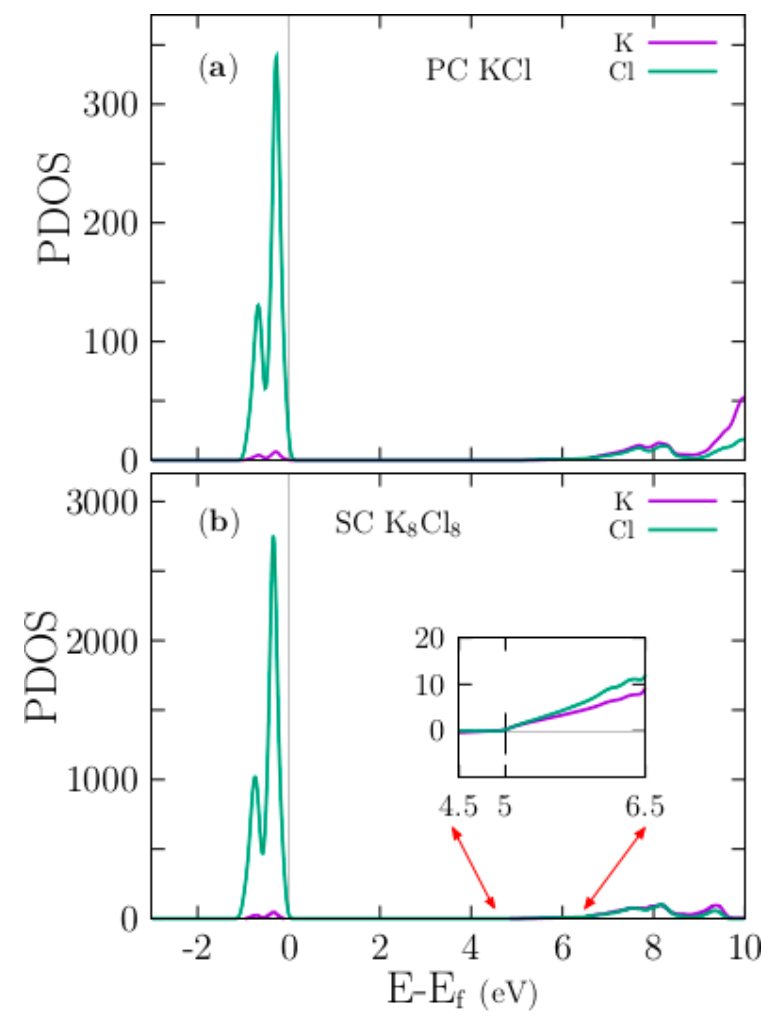

Figure 3. The upper panel (a) shows the Kohn-Sham projected density of states (PDOS) for the pristine $\mathrm{PC}$ for $\mathrm{KCl}$. The lower panel (b) shows the PDOS for the pristine $\mathrm{SC}$ of $\mathrm{K}_{8} \mathrm{Cl}_{8}$. In both plots, the Cl-3p states dominate at the Fermi level. 


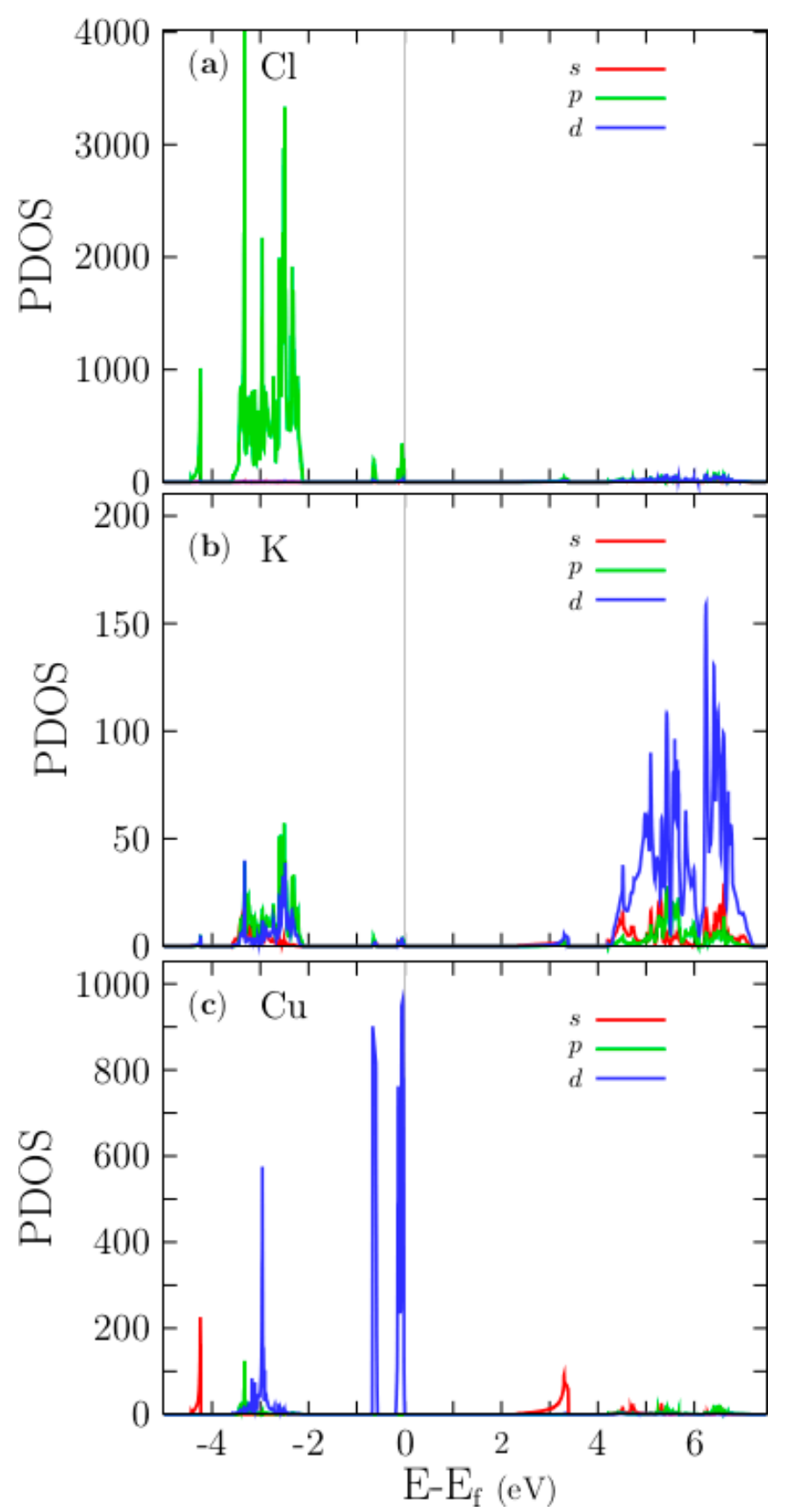

Figure 4. The upper panel (a): Kohn-Sham PDOS for the $\mathrm{Cl}$ atom. Middle panel (b): Kohn-Sham PDOS for the K atom. The lower panel (c): PDOS for the $\mathrm{Cu}$ atom. The $\mathrm{Cu}$-3d states dominate at the Fermi level with a sharply peaked density, and at $2.2 \mathrm{eV}$, the $\mathrm{Cu}-4 \mathrm{~s}$ states dominate. For all atoms, $\mathrm{s}^{-}, \mathrm{p}-$, and d-states are identified by red, green and blue lines, respectively.

\subsection{Folded Band Structure of the Doped $\mathrm{K}_{7} \mathrm{Cl}_{8}: \mathrm{Cu}$}

The effect of the $\mathrm{Cu}$ atom on the electronic properties was analyzed. Figure 5a shows the Kohn-Sham folded band structure for the doped $\mathrm{K}_{7} \mathrm{Cl}_{8}: \mathrm{Cu}$ system, along the same high-symmetry path used in the calculation for the pristine PC KCl. Figure 5b shows a zoomed view of the Kohn-Sham folded band structure in the range of -4.0 to $0.5 \mathrm{eV}$, where the dispersionless character of the bands located at the (shifted) Fermi level can be clearly observed. The lowest calculated direct Kohn-Sham bandgap is $2.2 \mathrm{eV}$ at the $\Gamma$ point. 


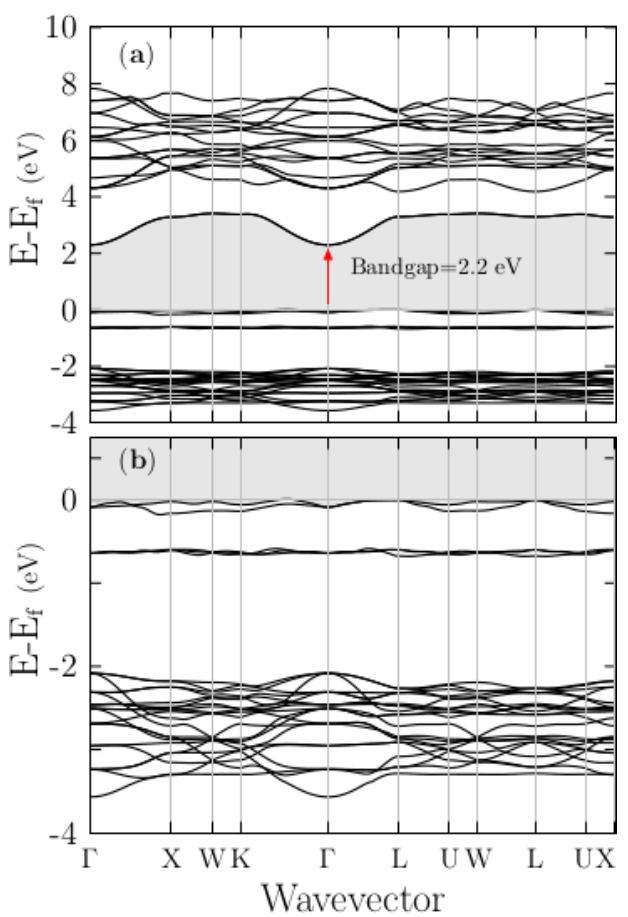

Figure 5. The upper panel (a) shows the calculated Kohn-Sham folded band structure of $\mathrm{SC} \mathrm{K}_{7} \mathrm{Cl}_{8}: \mathrm{Cu}$ for the case of Figure 1c and along the same high-symmetry directions of the BZ used in the PC. The lower panel (b) shows a zoomed region in the energy range of 4.0 to $0.5 \mathrm{eV}$. The lowest direct Kohn-Sham bandgap, indicated by the red arrow, is $2.2 \mathrm{eV}$ at the $\Gamma$ point.

PDOS is plotted in Figure 4a-c for $\mathrm{Cl}, \mathrm{K}$, and $\mathrm{Cu}$ atoms, respectively. $\mathrm{Cl}$ and $\mathrm{K}$ atoms present small p-state contributions at the Fermi level, as shown by Figure 4a,b. A closer examination of the PDOS for the $\mathrm{Cu}$ ion plotted in Figure 4c reveals two sharp peaks located around the Fermi level, the maxima of which are shifted by $\sim 0.6 \mathrm{eV}$ compared to one another. The two topmost valence bands are formed by $\mathrm{Cu}-3 \mathrm{~d}$ states and a couple of sub-bands, shown in Figure $5 \mathrm{~b}$, clearly localized at $0.6 \mathrm{eV}$ below the Fermi level. They are also composed of $\mathrm{Cu}-3 \mathrm{~d}$ states, as the PDOS indicates. In the energy range of -2.0 to $-4.0 \mathrm{eV}$, there are many bands bunched together with a dispersionless character, as is clearly shown in Figure 5. These bands are also mainly composed of Cl-3p states, as the PDOS reveals. In the energy range of 2 to $4 \mathrm{eV}$, there are also many bands bunched together with a dispersionless character. These bands are mainly composed of K-d states, as the PDOS reveals. The bottom conduction band also has a dispersionless character and is composed solely of $\mathrm{Cu}$-s states.

The panels (a-d) of Figure S6 and the panels (e-h) of Figure S7 shows the calculated Kohn-Sham folded band structure of $\mathrm{SC} \mathrm{K}_{7} \mathrm{Cl}_{8}: \mathrm{Cu}$ as a function of the doping site and along with the same high-symmetry directions of the BZ used in the PC; for the octahedral square bipyramidal shape case shown in Figure 1c and Figure S1a,h; and for slightly distorted twisted octahedra square bipyramidal shape cases shown in Figure S1b-g, respectively. The lowest direct Kohn-Sham bandgap of the KCl:Cu obtained is $3.2 \mathrm{eV}$ at the $\Gamma$ point, for the anisotropic case of Figure $\mathrm{S} 6 \mathrm{~b}$ as indicated by the red arrow and displayed in Table 2. (For the cases Figures S6b-d and S7e-g, the Kohn-Sham bandgap is 3.2 Ev. The anisotropy is indicative of the inhomogeneity in $\mathrm{KCl}: \mathrm{Cu}$ material; and causes significant changes in the electronic properties of $\mathrm{KCl}: \mathrm{Cu}$ system, opening the Kohn-Sham bandgap from $2.2 \mathrm{eV}$ to $3.3 \mathrm{eV}$. In contrast, it has little impact on the shape of the band structure keeping the dispersion on the first conduction band. Figures S6 and S7 show the Kohn-Sham folded band structure for all eight cases to facilitate the comparison.

To gain insight in the trend of the effect of $\mathrm{Cu}$ concentration in the electronic structure, we built the first and smallest cubic supercell that can be doped with $\mathrm{Cu}$ atom, with eight atoms basis $(\mathrm{Cu}, 3 \mathrm{~K}, 4 \mathrm{Cl})$, multiplicity 4 , shown in Figure $\mathrm{S} 2$ of the supplementary information. The density of the 
$\mathrm{Cu}$-doped $\mathrm{KCl}$ is $0.447 \mathrm{~g} / \mathrm{cm}^{3}$, considering the cubic SC with eight atoms. We show in Figure S8 the band structure calculated trough the k-path corresponding a cubic cell. The Kohn-Sham bandgap is $0.80 \mathrm{eV}$ at the $\Gamma$ point, and its character is indirect. Therefore, the energy bandgap decrease as the $\mathrm{Cu}$ electronic density increases. We can infer if $\mathrm{Cu}$ electronic density decreases, the band structure should increasingly resemble the pure $\mathrm{KCl}$ band structure.

The projected band structure (PBS) and PDOS of the doped $\mathrm{Cu} \mathrm{KCl}$ system are depicted in Figure 6. The PBS is color-coded in an informative manner: the color intensity corresponds to the degree of contribution of a particular orbital to the bands; thus visually, the contribution of orbitals to bands can be identified. The PBS of the $\mathrm{KCl}: \mathrm{Cu}$ system, (in Figure 6a) shows that the top-valence band, at the Fermi level and in the k-path line segments- $\Gamma \mathrm{W}, \mathrm{K}-\Gamma,-\mathrm{L}$, and $\mathrm{U}-\mathrm{X}$, is formed by the contribution of the $3 \mathrm{~d}$ states, and at the $\mathrm{L} \mathrm{k}$-points mainly by the $3 \mathrm{p}$-states. Indeed, Figure $4 \mathrm{c}$ indicates that the former contributions belong to the $\mathrm{Cu}$ atom and the latter to the chlorines atoms. The valence bands located at the energy range of -4 to $-2 \mathrm{eV}$ are mainly formed by $\mathrm{Cl}-3 \mathrm{p}$ states, as it is shown in Figure 6. On the other hand, the $4 \mathrm{~s}$ states of the $\mathrm{Cu}$ atom form the first conduction band at the K-G-L points. The color intensity of the first conduction band in the X-W-K and L-U-W-L-U-X segments indicates a mixture of $\mathrm{s}$ and $p$ states (hybridization). The higher-energy conduction bands (blue lines) are composed mainly of the $\mathrm{d}$ electronic-states from the $\mathrm{K}$ atoms. Since critical optical transitions take place at the $\Gamma$ point and involve only the top valence band and the first conduction band, a $3 \mathrm{~d}-4 \mathrm{~s}$ optical transition of the $\mathrm{Cu}$ must occur, this is calculated in the optical properties section.

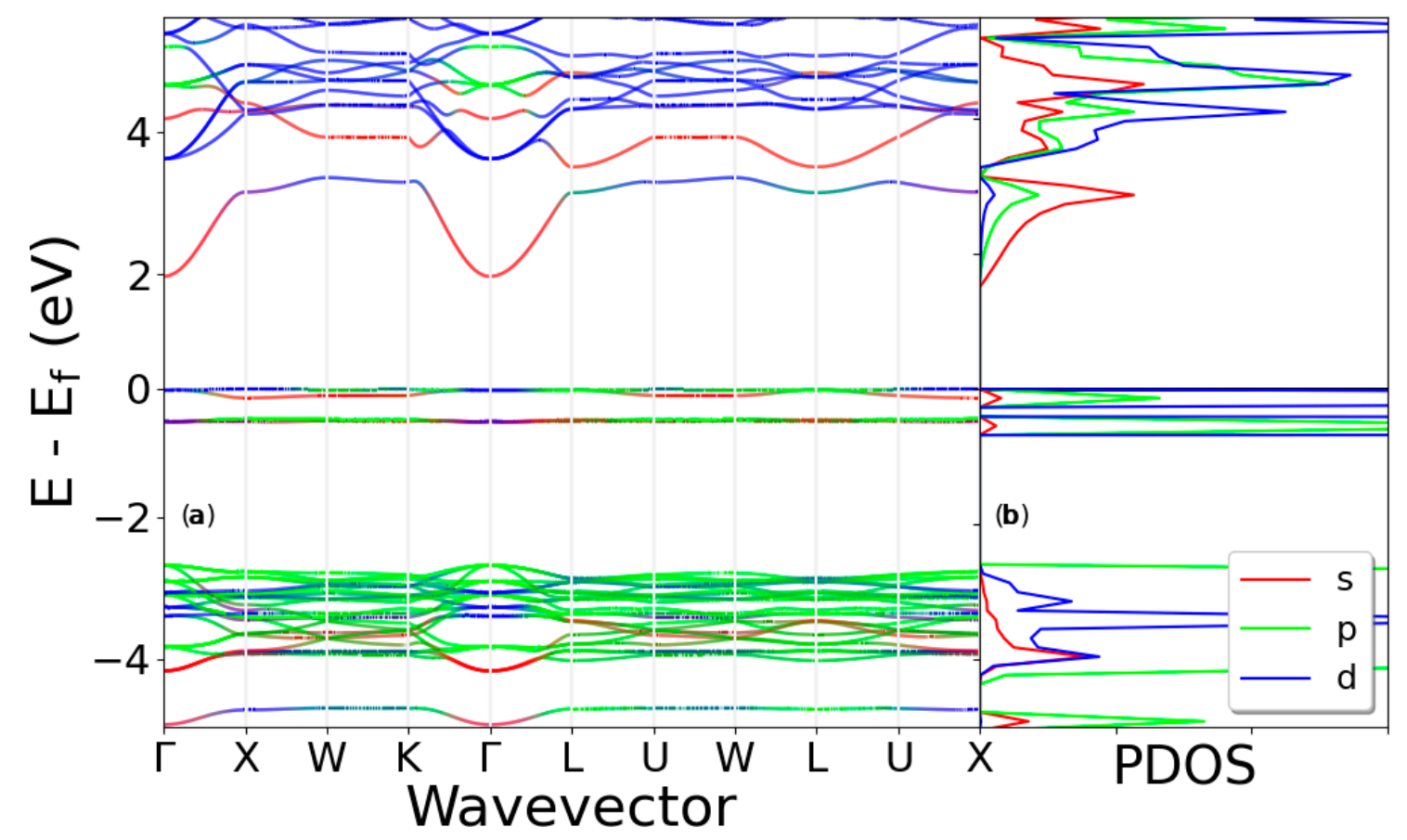

Figure 6. (Color online.) Panel (a) shows the Kohn-Sham projected band structure (PBS), and panel (b) shows the PDOS for the doped $\mathrm{K}_{7} \mathrm{Cl}_{8}: \mathrm{Cu}$ system, s-, p-, and d-states are identified by the red, green, and blue lines, respectively.

\subsection{Unfolded Band Structure of Pristine $\mathrm{K}_{8} \mathrm{Cl}_{8}$ and Doped $\mathrm{K}_{7} \mathrm{Cl}_{8}: \mathrm{Cu}$}

To gain a better understanding of the $\mathrm{Cu}$ atom doping effects on the $\mathrm{KCl}$ at the electronic level, we unfolded the band structure of the doped $\mathrm{SC}_{7} \mathrm{Cl}_{8}: \mathrm{Cu}$. To perform a test and make a comparison with the doped system, we also unfolded the pristine $\mathrm{SC} \mathrm{K}_{8} \mathrm{Cl}_{8}$. The in Figure $7 \mathrm{~b}$ shows the unfolded band structure for pristine $\mathrm{SC} \mathrm{K}_{8} \mathrm{Cl}_{8}$ or the so-called effective band structure (EBS), introduced by Popescu $[39,43]$ and other researchers $[42,49,75]$. The EBS can be directly compared to angle-resolved photoemission spectroscopy (ARPES) data [55]. The EBS for the pristine $\mathrm{SC} \mathrm{K}_{8} \mathrm{Cl}_{8}$ should give similar 
eigenvalues obtained from the band structure calculation employing $\mathrm{PC} \mathrm{KCl}$. We found the band structures of the PC and SC KCl to be identical due to the perfect translation of the primitive cells that built the pristine $\mathrm{SC} \mathrm{K}_{8} \mathrm{Cl}_{8}$. To facilitate mutual comparison, the EBS shown in Figure 7 was computed within the same energy range and high-symmetry directions in the BZ employed in the band structure (blue lines) of PC KCl, as shown in Figure 2a. The EBS of the doped $\mathrm{SC} \mathrm{K}_{7} \mathrm{Cl}_{8}: \mathrm{Cu}$ is depicted in the in Figure 7a. As shown in this figure, the effect of $\mathrm{Cu}$ doping on the electronic structure of pure $\mathrm{KCl}$ is dramatic. As shown in Figure $7 \mathrm{~b}$, the $\mathrm{Cu}$ doping greatly modifies the electronic structure of $\mathrm{KCl}$, narrowing the energy bandgap and opening small gaps in the valence and conduction bands. The Fermi level has shifted to zero energy axes.

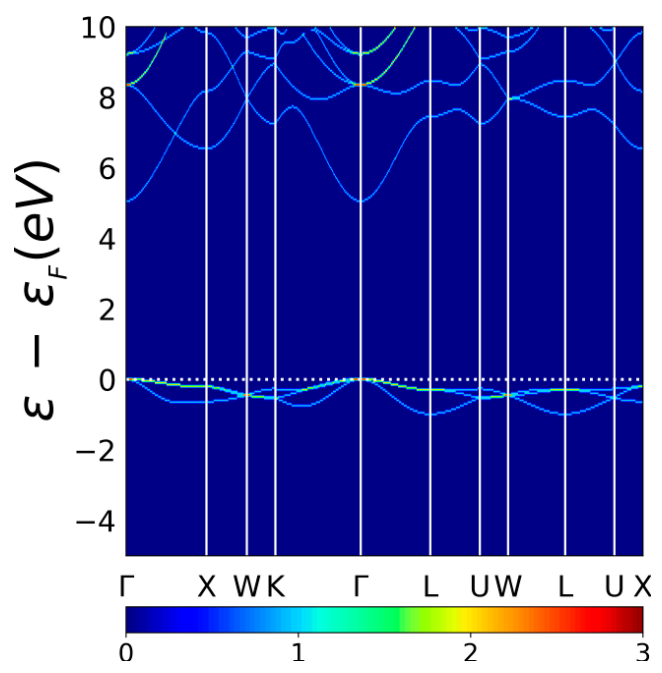

(a)

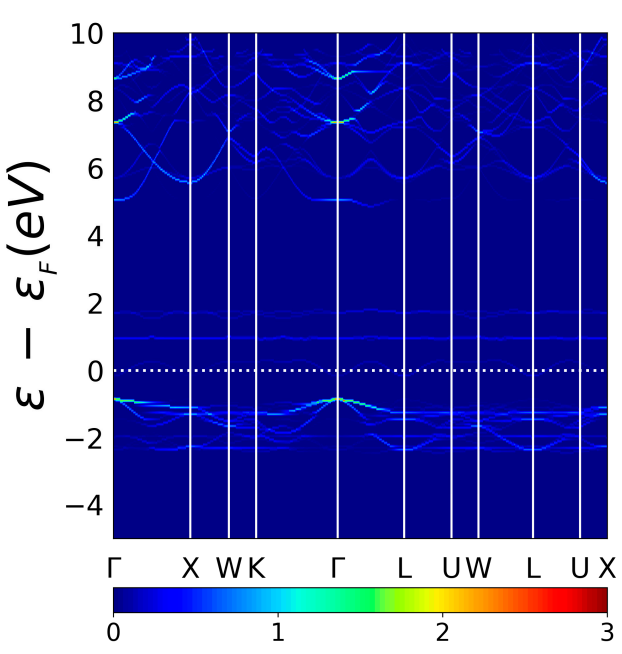

(b)

Figure 7. Effective band structure (EBS) for pristine $\mathrm{SC} \mathrm{K}_{8} \mathrm{Cl}_{8}(\mathbf{a})$ and $\mathrm{EBS}$ for doped $\mathrm{SC} \mathrm{K}_{7} \mathrm{Cl}_{8}: \mathrm{Cu}(\mathbf{b})$, along with the same high-symmetry directions in the $\mathrm{BZ}$ and the same energy range. The color scale represents the number of PC bands crossing the energy interval $(0.05 \mathrm{eV})$ at a given primitive wave vector $[41,51,76]$.

A detailed examination of Figure 7 indicates that $\mathrm{Cu}$-doping of the $\mathrm{KCl}$ structure reduces the direct energy bandgap and that the unfolding procedure preserves the same direct character of the energy bandgap (Figure 5). Previous studies suggest that the folded bands fail to reproduce the indirect bandgap character $[38,77]$. The cutoff of the energy bandgap is attributed to the appearance of empty bands with a dispersionless character located at 1.0 and $2.0 \mathrm{eV}$. The Figure $7 \mathrm{~b}$ shows the existence of a single valence band located at the Fermi level with small peaks and valleys; despite these, the band is quite flat, thus producing a pronounced peak in the PDOS. Further analysis of the valence bands reveals bunched-together bands in the energy range of -3.0 to $-1.0 \mathrm{eV}$, showing many small energy gap openings that are not observed in the folded band structure of the $\mathrm{SC} \mathrm{K}_{7} \mathrm{Cl}_{8}: \mathrm{Cu}_{\text {, }}$ shown in Figure 2b. (black lines) Interestingly, similar energy gap openings were found in bi-graphene studies [55]. Moreover, a small gap appeared in the bottom conduction bands at an energy of $5.0 \mathrm{eV}$, located at the $\Gamma-\mathrm{L}$ valley. A direct comparison between the bottom conduction band of pristine EBS (in Figure 7a) and the bottom conduction band of the doped EBS (in Figure 7b) shows that significant changes occurred in the $\mathrm{k}-\Gamma-\mathrm{L}$ valley, which drastically changed the band dispersion and opened small gaps, as mentioned before.

\subsection{Optical Properties}

Optical properties (OP) are a valuable source of atomic structural information, and their electronic band structure largely determines them $[78,79]$. Once we obtained the optimized crystal structure of the $\mathrm{KCl}$, the OPs were calculated by evaluating Equation (3). Figure 8a shows the $x x$ component of the 
imaginary $\left[\chi_{1}^{x x}(-\omega ; \omega)\right]$ part of the electric susceptibility tensor for the pristine $\mathrm{PC} \mathrm{KCl}$ in the energy range of 0 to $20 \mathrm{eV}$, which is in good agreement with previous calculations [28].

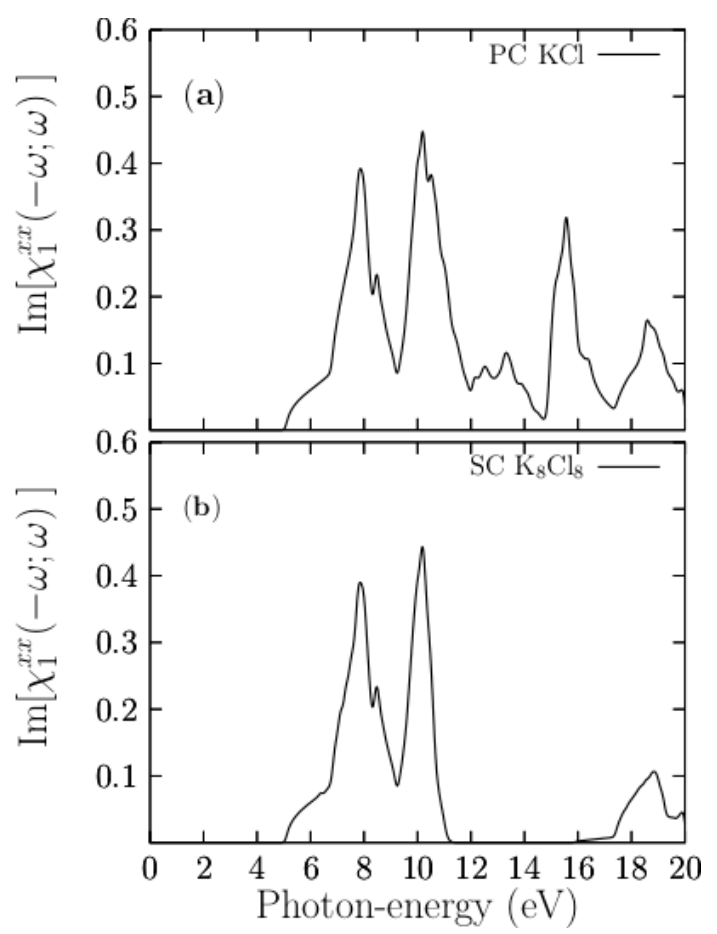

Figure 8. The upper panel (a) shows the calculated $\left[\chi_{1}^{x x}(-\omega ; \omega)\right]$ part corresponding to pristine PC $\mathrm{KCl}$, with the FCC structure and two atoms in the base. The lower panel (b) shows the calculated $\left[\chi_{1}^{x x}(-\omega ; \omega)\right]$ corresponding to pristine $\mathrm{SC} \mathrm{K}_{8} \mathrm{Cl}_{8},(2 \times 2 \times 2$ pristine $\mathrm{PC} \mathrm{KCl})$, with 16 atoms in the base.

Figure 8a shows the $\left[\chi_{1}^{x x}(-\omega ; \omega)\right]$ part of the pristine PC. As shown in this figure, the $\left[\chi_{1}^{x x}(-\omega ; \omega)\right]$ part starts to rise at $5.0 \mathrm{eV}$, and it has peaks located at 7.8, 10.2, 15.5, and $18.6 \mathrm{eV}$. There are minor peaks located at 8.4 and $13.3 \mathrm{eV}$. At the onset of the signal, there is a shoulder in the energy range of 5.0 to $6.7 \mathrm{eV}$, which is mainly due to the direct transitions from the topmost valence bands (highest-energy valence bands) at the $\Gamma$ point. As the crystal structure of $\mathrm{KCl}$ is completely isotropic, the $\mathrm{xx}, \mathrm{yy}$, and $\mathrm{zz}$ components are also isotropic. Hereafter, we only present the $\mathrm{xx}$ component, and the scissor correction of $3.53 \mathrm{eV}$ was applied at the end. Figure $8 \mathrm{~b}$ shows the $\left[\chi_{1}^{x x}(-\omega ; \omega)\right]$ part or the pristine $\mathrm{SC}_{8} \mathrm{Cl}_{8}$ that also start to raise at $5 \mathrm{eV}$ and peaks located at 7.8 and $10.2 \mathrm{eV}$. The main difference between the PC and SC responses is located above $12.0 \mathrm{eV}$ on the energy axis because the number of electronic bands necessary to converge the SC system is higher than that of the PC. Nonetheless, the signals are similar in the energy range of 5 to $10 \mathrm{eV}$.

Figure 9 shows the real $R\left[\chi_{1}^{x x}(-\omega ; \omega)\right]$ part of the pristine $\mathrm{PC} \mathrm{KCl}, \mathrm{SCK}_{8} \mathrm{Cl}_{8}$ and $\mathrm{K}_{7} \mathrm{Cl}_{8}: \mathrm{Cu}$ systems. The real $R\left[\chi_{1}^{x x}(-\omega ; \omega)\right]$ part provides information about the polarizability of the material. Figure $9 \mathrm{a}$ shows that the static value $\left[\chi_{1}^{x x}(-\omega ; \omega)\right]$ is $0.1\left(\chi_{1}^{x x}(0)=0.1\right)$ for pristine PC $\mathrm{KCl}$. From here, it starts to increase and reach a large peak with a value of 0.3 at $6.9 \mathrm{eV}$. After that, a rapid decrease is observed until it reaches zero. The negative values of the real $R\left[\chi_{1}^{x x}(-\omega ; \omega)\right]$ part are observed in the energy range of 10.28 to $14.43 \mathrm{eV}$ and above $15.5 \mathrm{eV}$. At $10.28 \mathrm{eV}$, the real part changes sign. The negative values of the real part are indicative of metallic behavior, whereas a change of sign in the real part indicates the occurrence of plasmonic resonances in the energy region where it crosses the energy axes with a positive slope. The real part for the pristine SC shows in Figure $9 \mathrm{~b}$ has a similar behavior. 


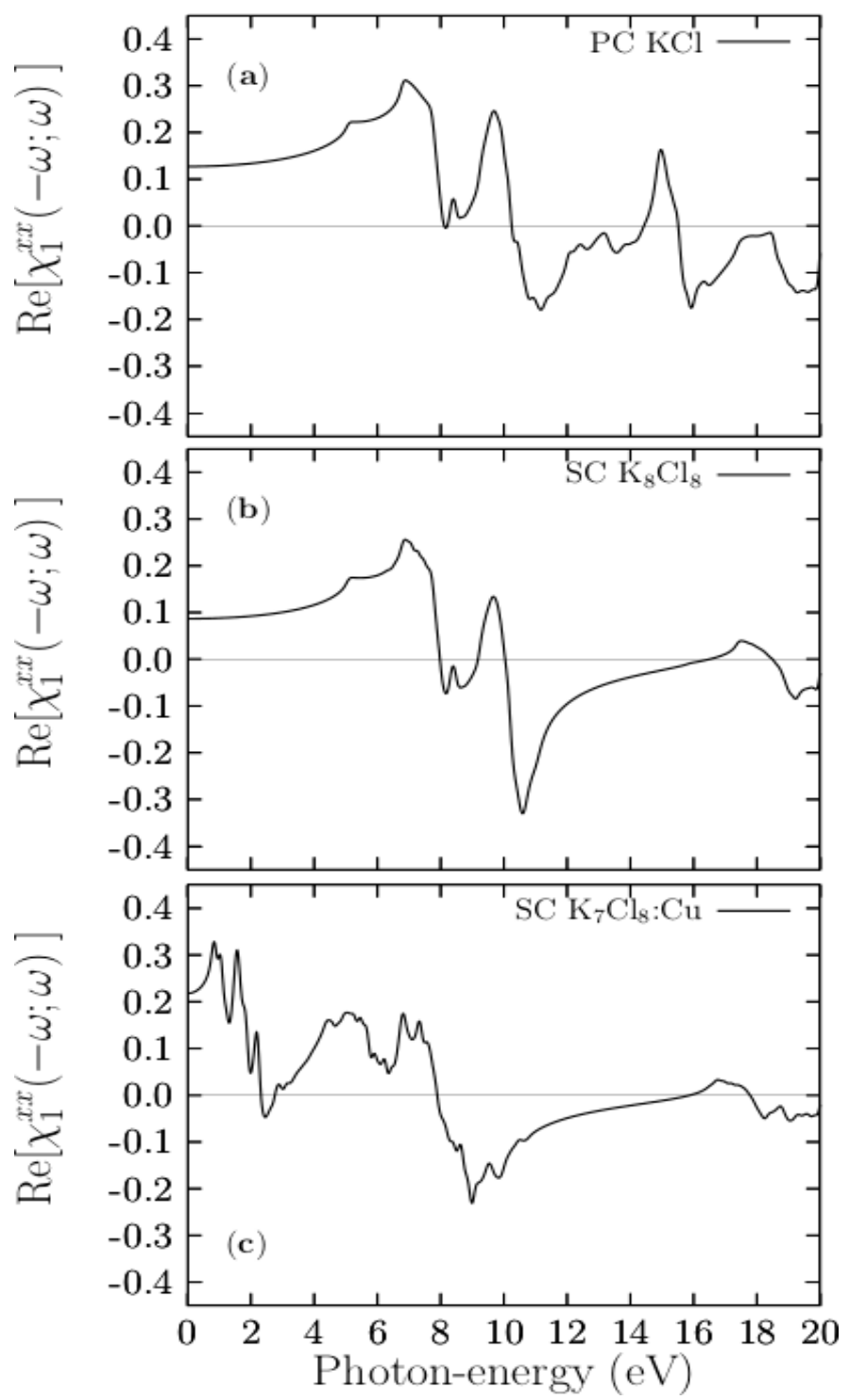

Figure 9. (Color online.) Upper panel (a) shows the calculated real $R\left[\chi_{1}^{x x}(-\omega ; \omega)\right]$ part of the PC KCl. Middle panel (b) shows the real $R\left[\chi_{1}^{x x}(-\omega ; \omega)\right]$ part for the pristine $\mathrm{SC} \mathrm{K}_{8} \mathrm{Cl}_{8}$ system. Lower panel (c) show the real $R\left[\chi_{1}^{x x}(-\omega ; \omega)\right]$ part of the doped $S C \mathrm{~K}_{7} \mathrm{Cl}_{8}$ :Cu system.

3.6. Breakdown of the $\left[\chi_{1}^{x x}(-\omega ; \omega)\right]$ Part of Pristine Supercell (SC) KCl into Different Band Contributions

Figure 10a shows the breakdown of the $\left[\chi_{1}^{x x}(-\omega ; \omega)\right]$ part, taking into account the transitions within an iterated cumulative sum (ICS) of all the valence bands, assigning to the top valence band the index $1\left(\mathrm{VB}_{1}\right)$, the sub-top valence band $2\left(\mathrm{VB}_{2}\right)$, and so on (e.g., $\left.\mathrm{VB}_{[1]}, \mathrm{VB}_{[1+2]}\right)$ against the single bottom conduction band $\left(\mathrm{CB}_{1}\right)$. In this in-house developed scheme, the first ICS corresponds to the contributions to the $\left[\chi_{1}^{x x}(-\omega ; \omega)\right]$ part due to the top valence and bottom conduction bands. The red line in Figure 10a clearly shows that the contribution to the onset of the signal up to $6.5 \mathrm{eV}$ is $50 \%$. The second ISC, which takes into account the top valence and sub-top valence bands against the single bottom conduction band, is depicted by the solid blue line in Figure 10a, which clearly shows that this ICS contributes to the $\left[\chi_{1}^{x x}(-\omega ; \omega)\right]$ part by almost $95 \%$ for the onset of the signal. 


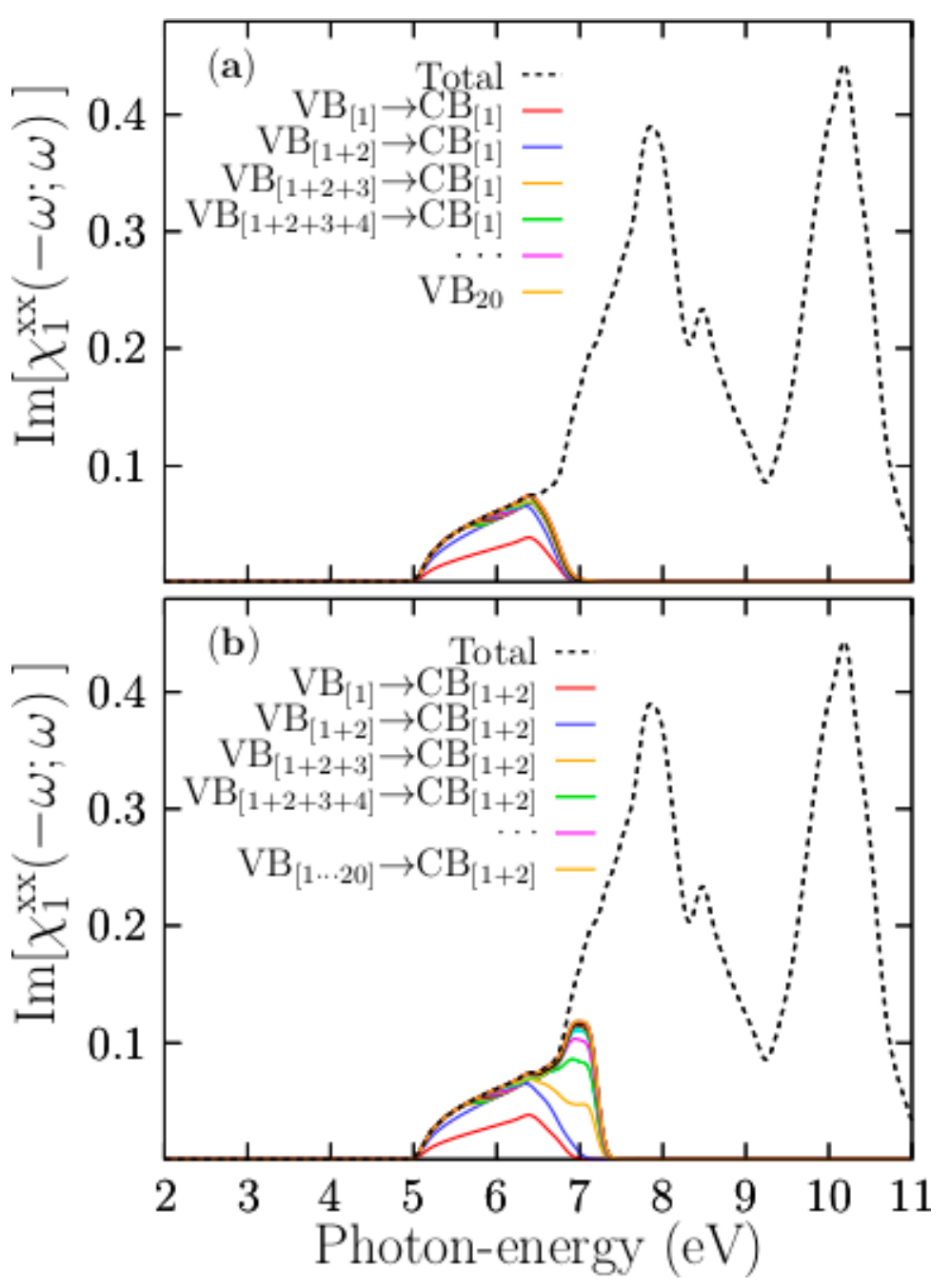

Figure 10. Breakdown of the imaginary part of the $\mathrm{SC} \mathrm{K}_{8} \mathrm{Cl}_{8}$ into different band contributions. The upper panel (a) shows the decomposition of the $\left[\chi_{1}^{\chi x}(-\omega ; \omega)\right]$ part by only considering the 20 highest valence band (VB) transitions to only the first conduction band (CB), e.g., $V B_{[1]} \rightarrow C, V B_{[1]+[2]} \rightarrow C B_{[1]}$, $V B_{[1]+[2]+[3]} \rightarrow C B_{[1]}$. The lower panel $(\mathbf{b})$ shows the decomposition of the $\left[\chi_{1}^{x x}(-\omega ; \omega)\right]$ part only considering the 20 highest VB transitions to only the first and second CBs, e.g., the red solid line is the $V B_{[1]} \rightarrow C B_{[1]+[2]}$. The suspension points, pink solid line, stand for terms not listed, but which follow the sequence. The black dotted line in both panels represents the $\chi_{1}^{x x}(-\omega ; \omega)$ part, taking into account all VBs and CBs considered in the calculation.

The rest of the ICS contributes only about $5 \%$. Twenty of the ICSs are plotted and shown in Figure 10a. Our calculated band contribution breakdown indicates that we only need the two topmost valence bands and the bottom conduction band to build up the onset of the signal of up to $6.5 \mathrm{eV}$ with an accuracy of $95 \%$. Moreover, based on the PDOS, the Cl-3p states mainly form the two topmost valence bands, and the bottom conduction band is composed of a mixture of Cl-s and K-s states. Therefore, the onset of the signal up to $6.5 \mathrm{eV}$ is due to the direct transitions involving Cl-3p, $\mathrm{Cl}-4 \mathrm{~s}$, and K-4s states at the $\Gamma$ point. Figure $10 \mathrm{~b}$ shows the breakdown of the $\left[\chi_{1}^{x x}(-\omega ; \omega)\right]$ part with an ICS considering all valence bands against the two bottom-most conduction bands. The figure shows that the contribution of the first ICS, depicted by the solid blue line, considers the transitions from the top valence band to the two bottom-most conduction bands, which are similar to those when we consider a single bottom conduction band. The third ICS, depicted as a solid yellow line in Figure 10b, builds the large peak located at 7.8 eV. In summary, and to solve the first large peak located at $7.8 \mathrm{eV}$ and peaks located at higher energies, we need to take higher conduction bands into account. 
3.7. The $\left[\chi_{1}^{x x}(-\omega ; \omega)\right]$ Part of the Doped $\mathrm{K}_{7} \mathrm{Cl}_{8}: \mathrm{Cu}$

The solid blue line shown in Figure 11a depicts the $\left[\chi_{1}^{x x}(-\omega ; \omega)\right]$ part that corresponds to the doped SC K7Cl8 octahedral square pyramidal shape shown in Figure 1c, the solid black line corresponds to the $\left[\chi_{1}^{x x}(-\omega ; \omega)\right]$ part of the pristine $\mathrm{SC} \mathrm{K}_{8} \mathrm{Cl}_{8}$ system. The solid red line corresponds to the doped $\mathrm{SC} \mathrm{K}_{7} \mathrm{Cl}_{8}: \mathrm{Cu}$ slightly distorted-twisted octahedra square bipyramidal shape shown in Figure S1b. We have included the three signals on the same graphic to compare them. We first focus on the isotropic case, solid blue line. Figure 11a shows that doping $\mathrm{KCl}$ with $\mathrm{Cu}$ had a drastic effect on in its optical properties. The main peak starts to rise at $0.9 \mathrm{eV}$ and ends at $4.0 \mathrm{eV}$ and is composed of four sub-peaks located at $1.1,1.87,2.3$, and $2.9 \mathrm{eV}$. In the energy range of 4.0 to $6.7 \mathrm{eV}$, there is a large peak located at $5.7 \mathrm{eV}$ with little ripples. Above the energy range of $6.7 \mathrm{eV}$, the signals and peaks for the doped and pristine systems are similar, and those peaks are located at an energy of $7.8 \mathrm{eV}$. The larger peak observed in the pristine signal around $10 \mathrm{eV}$, and not observed in the doped system, is caused by the number of conduction bands considered in the calculation. Figure $9 \mathrm{c}$ shows the $\left[\chi_{1}^{x x}(-\omega ; \omega)\right]$ part for the doped $\mathrm{SC} \mathrm{K}_{7} \mathrm{Cl}_{8}: \mathrm{Cu}$. It also clearly shows that the static value of the $\left[\chi_{1}^{x x}(-\omega ; \omega)\right]$ part is $0.22,\left(\chi_{1}^{x x}(0)=0.22\right)$, which is slightly more than double for the pristine case of 0.1 . As a consequence, the refraction index of the doped $\mathrm{SC} \mathrm{K}_{7} \mathrm{Cl}_{8}: \mathrm{Cu}$, which is related to the static value of the dielectric function, can increase at around $70 \%$ compared with the pristine case. Figure $11 \mathrm{~b}$ shows the $\left[\chi_{1}^{x x, y y, z z}(-\omega ; \omega)\right]$ part that corresponds to the doped SC $\mathrm{K}_{7} \mathrm{Cl}_{8}$ :Cu slightly distorted-twisted octahedral square bipyramidal shape shown in Figure S1b. The optical anisotropy is indicative of the inhomogeneous $\mathrm{KCl}: \mathrm{Cu}$, which results from the asymmetrically $\mathrm{Cu}-\mathrm{Cl}$ bonds in the octahedra of the unit cell. Figure S2b shows the imaginary part of the diagonal elements of the dielectric tensor. The solid red line, solid green line, and solid black line are the $\mathrm{xx}, \mathrm{yy}$, and zz elements. They reveal a considerable anisotropy in the energy range up to $3 \mathrm{eV}$. Beyond this point, the imaginary parts of the diagonal components of the dielectric tensor $\mathrm{xx}, \mathrm{yy}$, and $\mathrm{zz}$ are equal to each other; this is because the transitions contributing to this part of the spectrum come from the $\mathrm{Cl}$ and $\mathrm{K}$ atoms. At the onset of the signal (solid red line), the curve rapidly increases because the number of transitions contributing to the signal's onset comes from the $\mathrm{Cu}$ atoms. Thus, in the $\mathrm{x}$-axis direction, there is a larger concentration of $\mathrm{Cu}$ atoms than in the y-axis and $\mathrm{z}$-axis directions. The arrangement of six chlorine anions around the centered copper cation set a charge of -1 at each vertex, which causes the $\mathrm{d}-\mathrm{Cu}$ states to split into two groups with different energies. The energy difference between the two groups of orbitals is known as the crystal field splitting energy. On the other hand and from the electronic point of view, the magnitude of the splitting depends on the charge of the $\mathrm{Cu}$ atom, and a small change in the crystal field splitting energy is related to a distortion of the octahedron. The distorted-twisted octahedral square bipyramidal shape induces a difference in the crystal field splitting energy compared to that of the perfect octahedral square bipyramidal shape. So, the origin of the anisotropy comes from the difference in the crystal field-splitting energy between the distorted-twisted and the perfect octahedral square bipyramidal shapes. Moreover, the distorted system (Jahn-Teller effect) could be more stable than the undistorted one. In summary, the doping $\mathrm{Cu}$ atoms position has an enormous impact on the crystal field-splitting energy, and as a consequence, the optics and electronic properties change significantly. 


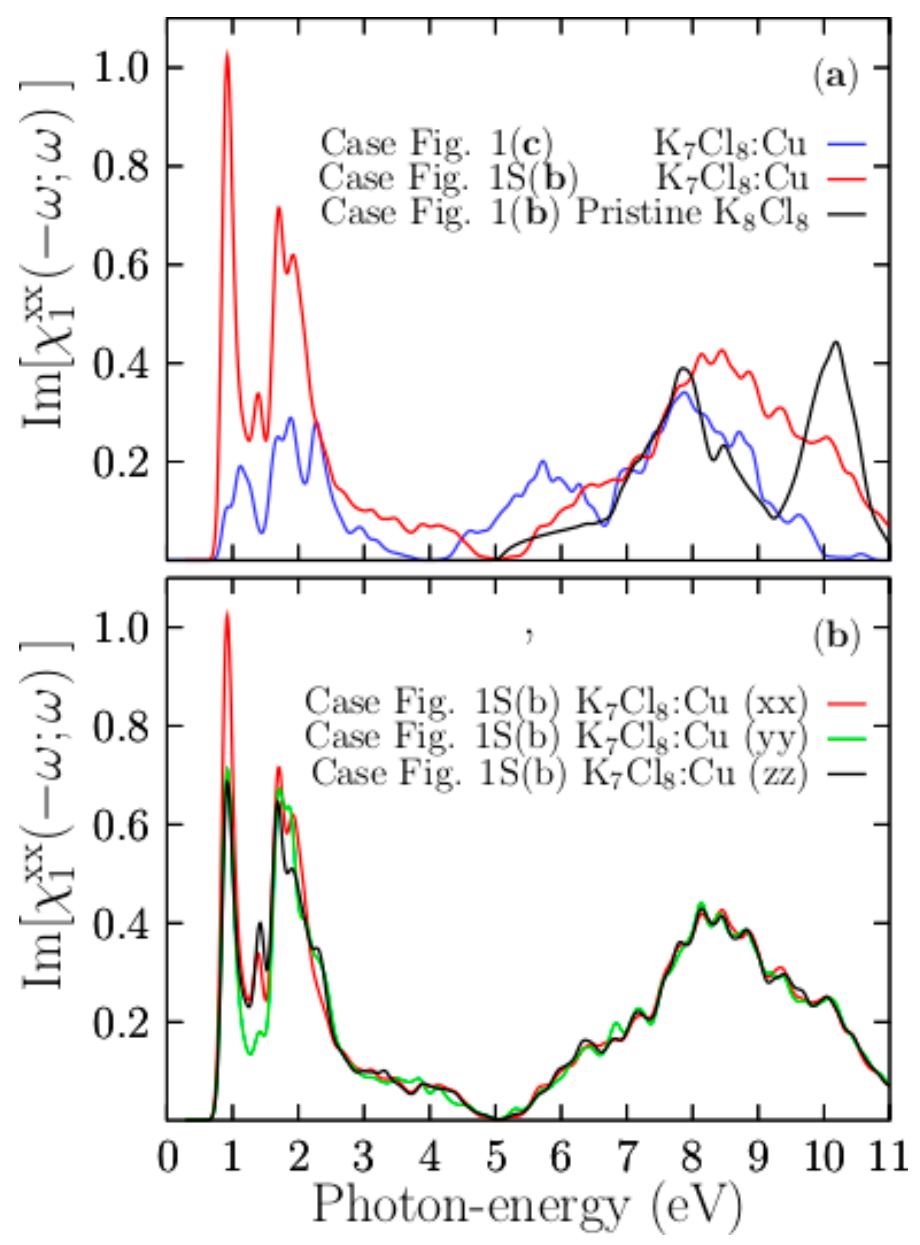

Figure 11. In the upper panel (a) the solid blue line represents the $\left[\chi_{1}^{x x}(-\omega ; \omega)\right]$ part of the $\mathrm{SC} \mathrm{K}_{7} \mathrm{Cl}_{8}: \mathrm{Cu}$ of the isotropic case shows in Figure 1c. The solid red line represent $\left[\chi_{1}^{x x}(-\omega ; \omega)\right]$ part of the SC $\mathrm{K}_{7} \mathrm{Cl}_{8}: \mathrm{Cu}$ of the anisotropic case is shown in Figure S1b. The solid black line represents the $\left[\chi_{1}^{x x}(-\omega ; \omega)\right]$ part of the pristine $\mathrm{SC} \mathrm{K}_{8} \mathrm{Cl}_{8}$ system. The lower panel (b) shows the $\left[\chi_{1}^{x x}(-\omega ; \omega)\right],\left[\chi_{1}^{y y}(-\omega ; \omega)\right]$, and $\left[\chi_{1}^{z z}(-\omega ; \omega)\right]$ parts of the $\mathrm{SC} \mathrm{K}_{7} \mathrm{Cl}_{8}: \mathrm{Cu}$, the octahedral square bipyramidal shape case is shown in Figure S1b. Notice the optical anisotropy is induced by the $\mathrm{Cu}$ doping position. The energy range is 0 to $11 \mathrm{eV}$.

Figure $9 \mathrm{c}$ also shows that the largest peak, with a value of 0.3 , is located at $0.8 \mathrm{eV}$, followed by a rapid decrease until 0.15 at $1.31 \mathrm{eV}$, after starting to rise to form a large peak located at $1.6 \mathrm{eV}$. From here, it begins to decrease until it crosses zero at $2.3 \mathrm{eV}$. At this energy value, the real part changes sign, indicating an occurrence of plasmonic resonances. Negative values of the $\left[\chi_{1}^{x x}(-\omega ; \omega)\right]$ part are observed in the energy range of 2.30 to $2.70,7.90$ to $15.9 \mathrm{eV}$, and above $17.8 \mathrm{eV}$. Comparing the $\left[\chi_{1}^{x x}(-\omega ; \omega)\right]$ part of the pristine and the $\left[\chi_{1}^{x x}(-\omega ; \omega)\right]$ part of the doped case, we find significant differences, similar to those found in the imaginary parts.

\subsection{Breakdown of the $\left[\chi_{1}^{x x}(-\omega ; \omega)\right]$ Part of the Doped SC $\mathrm{K}_{7} \mathrm{Cl}_{8}: \mathrm{Cu}$ into Band Contributions}

To elucidate the transitions contributing to the peaks that are located in the energy range of interest, we depict, in Figure 12, the computed breakdown of the $\left[\chi_{1}^{x x}(-\omega ; \omega)\right]$ part into band contributions (see also the video in the supplementary material). 


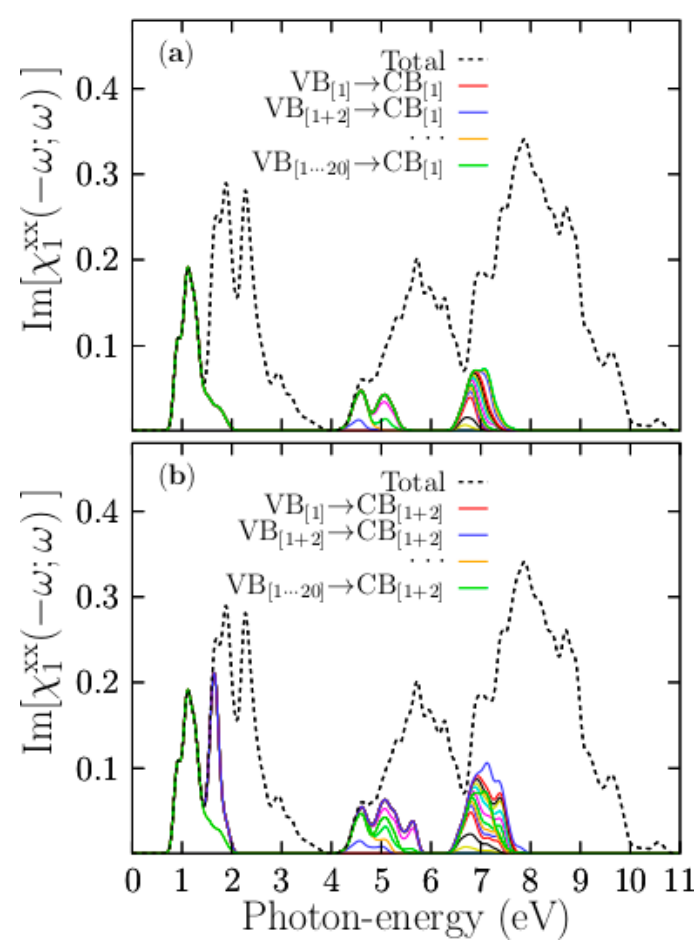

Figure 12. Breakdown of the $\left[\chi_{1}^{x x}(-\omega ; \omega)\right]$ part of the $\mathrm{K}_{7} \mathrm{Cl}_{8}: \mathrm{Cu}$ into different band contributions. The upper panel (a) shows the $\left[\chi_{1}^{x x}(-\omega ; \omega)\right]$ part, taking into account all valence bands with a single bottom conduction band. The lower panel $(\mathbf{b})$ shows the $\left[\chi_{1}^{x x}(-\omega ; \omega)\right]$ part, taking into account all valence bands with the two bottom-most conduction bands. The suspension points, yellow solid line, stand for terms not listed, but which follow the sequence. The black dotted line represents the total $\left[\chi_{1}^{x x}(-\omega ; \omega)\right]$ part, considering all valence and conduction bands available.

In Figure 12a, the first ICS that involves the single top valence band $\left(\mathrm{VB}_{[1]}\right)$ and single bottom conduction band $\left(\mathrm{CB}_{[1]}\right)$ accounts for the entire peak of the $\left[\chi_{1}^{x x}(-\omega ; \omega)\right]$ part located at $1.1 \mathrm{eV}$. Following the calculated PDOS presented in Figure 4c, the top valence band is dispersionless and is composed of $\mathrm{Cu}-3 \mathrm{~d}$ states, while the bottom conduction band is mainly composed of Cu-s states. Thus, the first peak of the $\left[\chi_{1}^{x x}(-\omega ; \omega)\right]$, located at $1.1 \mathrm{eV}$, is caused by the transitions of $\mathrm{Cu}-3 \mathrm{~d}$ to $\mathrm{Cu}-4 \mathrm{~s}$, mainly at the $\Gamma$ point. For $\mathrm{Cu}$-s states at around $2.2 \mathrm{eV}$, this figure also shows a PDOS with a low density of states, implying a broader bottom conduction band. In contrast, the same PDOS shows a very high density of states located at the Fermi level, indicating a relatively narrow valence band. Our calculations show that the intraband transitions take place from a dispersionless band composed of $\mathrm{Cu}-3 \mathrm{~d}$ states to the bottom conduction band formed by $\mathrm{Cu}-4 \mathrm{~s}$ states. Our calculated Kohn-Sham bandgap for the pristine case is $5.07 \mathrm{eV}$, which is an underestimation of $41 \%$ compared with the experimental value of $8.6 \mathrm{eV}$. If we consider this $59 \%$ to estimate the correct bandgap of the doped system, we obtain $3.90 \mathrm{eV}$; therefore, the first peak of the $\left[\chi_{1}^{x x}(-\omega ; \omega)\right]$ should be located at $3.17 \mathrm{eV}$, in excellent agreement with previous works [23]. Experimentally, it has been corroborated that there is a transition for copper, located at $2.2 \mathrm{eV}$, which explains why the copper has a reddish color. [80] Considering our calculations and the shift to $3.17 \mathrm{eV}$ of the first absorption peak, the doped system should have a violet color due to the transitions between the top valence band, formed by $\mathrm{Cu}-3 \mathrm{~d}$ states, and the bottom conduction band, formed by $\mathrm{Cu}-4 \mathrm{~s}$ states. The second ICS that involves the topmost and second-topmost valence bands $\left(\mathrm{VB}_{[1+2]}\right)$ and the single bottom conduction band $\left(\mathrm{CB}_{[1]}\right)$ is depicted in the solid blue line of Figure 10b. This transition contributes to the first peak located at $1.1 \mathrm{eV}$ and the peak at $4.3 \mathrm{eV}$. Therefore, the peak located at $5.7 \mathrm{eV}$ is also due to the $\mathrm{Cu}-3 \mathrm{~d}$ states. The ICS involving valence bands with 3 to 5 indices account for the small peaks located at $5.7 \mathrm{eV}$, and those that involve valence bands with 7 to 40 indices against the single bottom conduction band contribute to the most 
significant peak, located at $7.8 \mathrm{eV}$. According to the calculated PDOS shown in Figure 4c, these valence bands are located in the energy range of -2.0 to $-4.0 \mathrm{eV}$ and are mainly composed of Cl-3p states. This peak begins with transitions from $\mathrm{Cl}-3 \mathrm{p}$ states to the bottom conduction band, which is composed of a mixture of $\mathrm{Cu}-4 \mathrm{~s}$.

To gain more insight into the optical absorption details, we now break down the $\left[\chi_{1}^{x x}(-\omega ; \omega)\right]$ part considering the two bottom-most conduction bands against all the valence bands. The first ICS transition, taking into account the single top valence and two bottom conduction bands, is depicted as a solid red line in Figure 11b. It provides the sole contribution to the first peak of the part located at $1.1 \mathrm{eV}$ and contributes in part to a peak located at $1.87 \mathrm{eV}$. The behavior is similar to the case when we take a single bottom conduction band. Indeed, the four peaks of the $\left[\chi_{1}^{x x}(-\omega ; \omega)\right]$ part located at 1.1, 1.87, 2.3, and $2.9 \mathrm{eV}$ involve transitions from the two topmost valence bands to the bottom conduction bands. The PDOS indicates that those transitions involve $\mathrm{Cu}-3 \mathrm{~d}$ and K-3d states. In summary, the first peak located at $1.1 \mathrm{eV}$ is initiated by the transitions between the top valence bands, formed by pure $\mathrm{Cu}-3 \mathrm{~d}$ states, to the bottom conduction band, formed by $\mathrm{Cu}-4 \mathrm{~s}$ states. The second peak, located at $1.87 \mathrm{eV}$, is associated with transitions from the topmost valence bands to the second bottom bands. Therefore, it is contributed to by transitions from the top valence band formed by pure $\mathrm{Cu}-3 \mathrm{~d}$ states to the second conduction band formed by K-3d states (see Figure 4). The onset of the peak located at $5.7 \mathrm{eV}$ is initiated by the transitions between bands formed mainly of Cl-3p states to conduction bands formed of $\mathrm{Cu}-4 \mathrm{~s}$. Indeed, transitions to conduction bands formed by K-3d states (located at $4 \mathrm{eV}$ ) must also occur to form this peak.

\section{Conclusions}

In summary, we employed the band structure unfolding technique to study the electronic properties and break down the imaginary part of the dielectric susceptibility of the pristine $\mathrm{KCl}$ and doped $\mathrm{KCl}: \mathrm{Cu}$ systems to gain fundamental insights into the effect of copper doping on the electronic structure and optical properties of pure $\mathrm{KCl}$. Additionally, we considered different doping positions, where the $\mathrm{Cu}$ dopant occupies all the substitutional sites replacing host K cations. Substitutional doping leads to the distortion of the atomic structure near impurity atoms. The arrangement of six chlorine anions around the centered copper cation sets a charge of -1 at each vertex, which causes the $\mathrm{d}-\mathrm{Cu}$ states to split into two groups with different energies. The distorted-twisted octahedral square bipyramidal shape induces a difference in the crystal field-splitting energy compared to that of the perfect octahedral square bipyramidal shape. Therefore, the origin of the anisotropy comes from the difference in the crystal field-splitting energy between the distorted-twisted and the perfect octahedral square bipyramidal shapes. Moreover, the distorted system (Jahn-Teller effect) could be more stable than the undistorted one. The doping $\mathrm{Cu}$ atom's position has an enormous impact on the crystal field splitting and as a consequence, the optics and electronic properties change significantly. To study the changes in the band structure, we performed a direct comparison with the band structure of the pure $\mathrm{KCl}$. In addition, we unfolded the band structure of the pure $\mathrm{SC} \mathrm{KCl}$, exactly recovering the band structure of the PC $\mathrm{KCl}$ and analyzed the projected band structure of the doped $\mathrm{KCl}: \mathrm{Cu}$ Our findings show significant differences between the unfolded band structure of the $\mathrm{Cu}$-doped $\mathrm{KCl}$ and the unfolded band structure of pure $\mathrm{KCl}$. For the doped system, the bandgap was drastically reduced. Moreover, a dispersionless electronic band associated to $\mathrm{Cu}-3 \mathrm{~d}$ states appears at the Fermi level, and the conduction bands above the Fermi level are attributed to the $\mathrm{Cu}-4$ s electronic states. There is also less dispersion in the bottom conduction band of the doped system and a large dispersion in the bottom conduction band for pristine $\mathrm{KCl}$. In general, the main differences between the folded and the unfolded band structures of the doped $\mathrm{KCl}$ are the drastic change in the overall dispersion and the opening of small energy gaps in the bands, which were revealed by the unfolding procedure. The unfolding method was proven useful in the study of doped materials or those containing vacancies. The projected band structure shows that there are $\mathrm{Cu}-3 \mathrm{~d}$ states at the $\Gamma$ point that belongs to the top valence band; also there are $\mathrm{Cu}-4 \mathrm{~s}$ states in $\Gamma$ the point, that belong to the first conduction band; as a consequence all the transitions that 
take place at the $\Gamma$ point and between top valence and first conduction bands are transitions $3 \mathrm{~d}-4 \mathrm{~s}$ of the $\mathrm{Cu}$ atom. To gain insight in the effect of $\mathrm{Cu}$ concentration in the electronic structure, we built the first and smallest cubic supercell that can be doped with a $\mathrm{Cu}$ atom and calculated the folded band structure. We found that the Kohn-Sham bandgap is $0.80 \mathrm{eV}$ and its character is indirect. The trend is that the energy bandgap decrease as the $\mathrm{Cu}$ electronic density increases and vice versa. To study the optical properties, we broke down the $\left[\chi_{1}^{x x}(-\omega ; \omega)\right]$ part into band contributions. The sum-over-states formalism allows one to decompose band-to-band contribution through partial band-index summation, the bands of which significantly contribute to $\left[\chi_{1}^{x x}(-\omega ; \omega)\right]$. This methodology, proposed here together with PDOS, allows us to identify and analyze the origin of the peaks presented in the $\left[\chi_{1}^{x x}(-\omega ; \omega)\right]$ part. Our findings for the doped case show that the first peak of $\left[\chi_{1}^{x x}(-\omega ; \omega)\right]$ located at $1.1 \mathrm{eV}$ is due to transitions between the top valence band, which consists solely of Cu-3d states, to the bottom conduction band, formed of $\mathrm{Cu}-4 \mathrm{~s}$ states. Therefore, the first peak is attributed to the transitions from $\mathrm{Cu}-3 \mathrm{~d}$ to $\mathrm{Cu}-4 \mathrm{~s}$ states. The second peak, located at $1.87 \mathrm{eV}$, is due to transitions between the top valence band and bottom conduction band. In addition, transitions between the top valence band and the sub-bottom conduction band were ascribed to K-3d states, i.e., the second peak of $\left[\chi_{1}^{x x}(-\omega ; \omega)\right]$ located at $1.87 \mathrm{eV}$ is due to an admixture of electronic transitions from $\mathrm{Cu}-3 \mathrm{~d}$ to $\mathrm{Cu}-4$ s states and from $\mathrm{Cu}-3 \mathrm{~d}$ to K-3d states. We explain the origin of the onset of the signals and demonstrate that the methodology of decomposing the imaginary part into band contributions is a powerful tool in achieving a better understanding of the transitions participating in the optical response peaks.

Supplementary Materials: The following are available online at http://www.mdpi.com/1996-1944/13/19/4300/s1, Figure S1: Supercell of KCl doped with Cu atom, Figure S2: Cubic supercell of pure KCl with eight atoms at the base, Table S1: List of non-equivalent atoms in the unit cell, Table S2: List of atoms in the unit cell, Table S3: Lattice vectors, Figure S3: The octahedral square bipyramidal shape, Figure S4: The scissors correction in band structure PC and SC, Figure S5: The scissors corrected projected density of states in pure KCl, Figure S6: Kohn-Sham folded band structure, doping position (a-d) Figure S7: Kohn-Sham folded band structure, doping position (e-h), Figure S8: the folded bandstructure of the $\mathrm{Cu}$ doped supercell for $\mathrm{KCl}\left(\mathrm{CuK}_{3} \mathrm{Cl}_{4}\right)$, Video S1: band-to-band contribution to dielectric function.

Author Contributions: C.C.-Q. performed the calculations and drafted the manuscript; J.L.C. software design and development, performed calculations and analysis, drafted the manuscript, contributed to the conception of the study, performed the data analyses, and revised the manuscript. R.A. contributed to the conception of the study and drafted the manuscript; R.N.-G. performed calculations and drafted the manuscript; A.P.-A. contributed to the conception of the study, performed the data analyses, and revised the manuscript. All authors have read and agreed to the published version of the manuscript.

Funding: This research was funded by Consejo Nacional de Ciencia y Tecnología (CONACYT), Mexico (grant number A1-S-39326).

Acknowledgments: Computational resources supporting this work were provided by ACARUS through the High-Performance Computing Area of the University of Sonora.

Conflicts of Interest: The authors declare no conflict of interest.

\section{References}

1. Bhandari, K.P. Optical properties of alkali halides in ultraviolet spectral regions. Optics 2019, 1, 18-31. [CrossRef]

2. Baldochi, S.; Ranieri, I. Alkali halide crystals growth. In Reference Module in Materials Science and Materials Engineering; Elsevier: Amsterdam, The Netherlands, 2016; Volume 657, pp. 74-78.

3. Sirdeshmukh, D.B.; Sirdeshmukh, L.; Subhadra, K.G. Alkali Halides: A handbook of Physical Properties; Springer: Berlin/Heidelberg, Germany, 2001; Volume 49, pp. 1-281. [CrossRef]

4. Gopikrishnan, C.R.; Jose, D.; Datta, A. Electronic structure, lattice energies and born exponents for alkali halides from first principles. AIP Adv. 2012, 2, 012131. [CrossRef]

5. Zhang, C.; Andersson, T.; Svensson, S.; Bjorneholm, O.; Huttula, M.; Mikkela, M.H.; Tchaplyguine, M.; Ohrwall, G. Ionic bonding in free nanoscale $\mathrm{NaCl}$ clusters as seen by photoelectron spectroscopy. J. Chem. Phys. 2011, 134, 124507. [CrossRef] [PubMed]

6. Chen, Z.; Xiao, H.; Zu, X. First Principles Study of Structural, Electronic and Optical Properties of $\mathrm{KCl}$ Crystal. Chem. Phys. 2006, 330, 1-8. [CrossRef] 
7. Cepanec, K.; Vugrinec, S.; Cvetkovic, T.; Ranilovic, J. Potassium Chloride-Based Salt Substitutes: A Critical Review with a Focus on the Patent Literature. Compr. Rev. Food Sci. Food Saf. 2017, 16, 881-894. [CrossRef]

8. Palik, E. Handbook of Optical Constants of Solids. In Handbook of Thermo-Optic Coefficients of Optical Materials with Applications; Elsevier Science: Amsterdam, The Netherlands, 1997.

9. Feridoun, S.; Ebrahim, H.A.; Somayeh, S.P.; Taravati, A. $\mathrm{KCl}$ single crystals growth with $\mathrm{Mn}, \mathrm{Ag}$ and in impurities by Czochralski method and study of impurities influence on their properties. Open J. Phys. Chem. 2012, 2, 185-188. [CrossRef]

10. Polosan, S.; Tsuboi, T.; Apostol, E.; Topa, V. Electrolytic reduction of Tl+ ions in $\mathrm{KCl}$ crystals. Opt. Mater. 2007, 30, 95-97. [CrossRef]

11. Shiehpour, M.; Solgi, S.; Tafreshi, M.J. ZnO-doped $\mathrm{KCl}$ single crystal with enhanced UV emission lines. Appl. Phys. A 2019, 125, 1-9. [CrossRef]

12. Tomiki, T. Optical Constants and Exciton States in $\mathrm{KCl}$ Single Crystals. II. The Spectra of Reflectivity and Absorption Constant. J. Phys. Soc. Jpn. 1967, 23, 1280-1296. [CrossRef]

13. Baldini, G.; Bosacchi, B. Optical properties of alkali-halide crystals. Phys. Rev. 1968, 166, 863-870. [CrossRef]

14. Baldini, G.; Bosacchi, A.; Bosacchi, B. Exciton-phonon interaction in alkali halides. Phys. Rev. Lett. 1969, 23, 846-848. [CrossRef]

15. Said, K.I.; Green, G.W. Optical properties of caesium iodide in the vacuum ultraviolet. J. Phys. C Solid State Phys. 1977, 10, 479-488. [CrossRef]

16. Philipp, H.R.; Ehrenreich, H. Intrinsic optical properties of alkali halides. Phys. Rev. 1963, 131, $2016-2022$. [CrossRef]

17. Blechschmidt, D.; Klucker, R.; Skibowski, M. Dielectric properties of $\mathrm{KCl}, \mathrm{KBr}$, and $\mathrm{KI}$ single crystals in the extreme ultraviolet up to $35 \mathrm{eV}$. Phys. Status Solidi 1969, 36, 625-634. [CrossRef]

18. Kondo, S.I.; Nakamura, K. Strain effects on the fundamental absorption in alkali iodides. J. Phys. Soc. Jpn. 1970, 28, 1381. [CrossRef]

19. Erdinc, B.; Secuk, M.N.; Aycibin, M.; Gülebagan, S.E.; Dogan, E.K.; Akkus, H. Ab-initio calculations of physical properties of alkali chloride $\mathrm{XCl}(\mathrm{X}=\mathrm{K}, \mathrm{Rb}$ and $\mathrm{Li})$ under pressure. Comput. Condens. Matter 2015, 4, 6-12. [CrossRef]

20. Shamp, A.; Saitta, P.; Zurek, E. Theoretical predictions of novel potassium chloride phases under pressure. Phys. Chem. Chem. Phys. 2015, 17, 12265-12272. [CrossRef]

21. Aguado, A. Ga+, In+, and Tl+ impurities in alkali halide crystals: Distortion trends. J. Chem. Phys. 2000, 113, 8680-8685. [CrossRef]

22. Bouhdjer, L.; Addala, S.; Chala, A.; Halimi, O.; Boudine, B.; Sebais, M. Elaboration and characterization of a $\mathrm{KCl}$ single crystal doped with nanocrystals of $\mathrm{a} \mathrm{Sb}_{2} \mathrm{O}_{3}$ semiconductor. J. Semicond. 2013, 34, 043001. [CrossRef]

23. Myasnikova, A.; Mysovsky, A.; Paklin, A.; Shalaev, A. Structure and optical properties of copper impurity in $\mathrm{LiF}$ and NaF crystals from ab initio calculations. Chem. Phys. Lett. 2015, 633, 218-222. [CrossRef]

24. Daniel, D.J.; Ramasamy, P.; Madhusoodanan, U. Optical properties and irradiation effects of $\mathrm{Cu}+$ and Eu2+ doped alkali halide single crystals grown from melt using Czochralski technique. Optik 2013, 124, 1466-1468. [CrossRef]

25. Winter, N.W.; Pitzer, R.M.; Temple, D.K. Theoretical study of a Cu+ ion impurity in a NaF host. J. Chem. Phys. 1987, 86, 3549-3556. [CrossRef]

26. Preto, P.D.; Balraj, V.; Dhabekar, B.S.; Watanabe, S.; Rao, T.G.; Cano, N.F. Synthesis, thermoluminescence, defect center and dosimetric characteristics of LiF: Mg, Cu, P, Si phosphor. Appl. Radiat. Isot. 2017, 130, 21-28. [CrossRef] [PubMed]

27. Preto, P.D.; Balraj, V.; Dhabekar, B.S.; Watanabe, S.; Rao, T.G. Synthesis, thermoluminescence, defect centers and dosimetric characteristics of LiF:Mg,Cu,B phosphor. Appl. Radiat. Isot. 2016, 118, 95-101. [CrossRef] [PubMed]

28. Núñez-González, R.; Aceves, R.; Cabellos, J.L.; Posada-Amarillas, A. Effect of substitutional Cu atoms on the electronic and optical properties of KCl: A DFT approach. Mater. Today Commun. 2020, 22, 100831. [CrossRef]

29. Goldberg, A.; McClure, D.; Pedrini, C. Optical absorption and emission spectra of Cu+: NaF single crystals. Chem. Phys. Lett. 1982, 87, 508-511. [CrossRef]

30. Uhl, E.; Leitão, A.A.; Rocha, A.B. Transition energies and oscillator strength calculated for d-s symmetry-forbidden electronic transition for $\mathrm{Cu}+$ impurities in sodium fluoride host lattice. Chem. Phys. 2011, 389, 102-106. [CrossRef] 
31. Sun, Y.; Zhang, J.; Ding, J.; Yan, X. First-principles study on electronic and optical properties of Cu-doped LiF with Li vacancy. Phys. B Condens. Matter 2012, 407, 2458-2461. [CrossRef]

32. Cabellos, J.L.; Mendoza, B.S.; Escobar, M.A.; Nastos, F.; Sipe, J.E. Effects of nonlocality on second-harmonic generation in bulk semiconductors. Phys. Rev. B 2009, 80, 155205. [CrossRef]

33. Walker, D.; Verma, P.; Cranswick, L.; Jones, R.; Clark, S.; Buhre, S. Halite-sylvite thermoelasticity. Am. Mineral. 2004, 89. [CrossRef]

34. Schwabegger, G.; Djuric, T.; Sitter, H.; Resel, R.; Simbrunner, C. Morphological and structural investigation of sexithiophene growth on $\mathrm{KCl}$ (100). Cryst. Growth Des. 2013, 13, 536-542. [CrossRef] [PubMed]

35. Li, J.; Duan, C.g.; Gu, Z.q.; Wang, D.s. Linear optical properties and multiphoton absorption of alkali halides calculated from first principles. Phys. Rev. B 1998, 57, 2222-2228. [CrossRef]

36. Aroyo, M.I.; Kirov, A.; Capillas, C.; Perez-Mato, J.M.; Wondratschek, H. Bilbao Crystallographic Server. II. Representations of crystallographic point groups and space groups. Acta Crystallogr. Sect. A 2006, 62, 115-128. [CrossRef] [PubMed]

37. Setyawan, W.; Curtarolo, S. High-throughput electronic band structure calculations: Challenges and tools. Comput. Mater. Sci. 2010, 49, 299-312. [CrossRef]

38. Cartoixa, X.; Palummo, M.; Hauge, H.I.T.; Bakkers, E.P.A.M.; Rurali, R. Optical emission in hexagonal SiGe nanowires. Nano Lett. 2017, 17, 4753-4758. [CrossRef]

39. Popescu, V.; Zunger, A. Extracting E versus $\sim \mathrm{k}$ effective band structure from supercell calculations on alloys and impurities. Phys. Rev. B 2012, 85, 085201. [CrossRef]

40. Maspero, R.; Sweeney, S.J.; Florescu, M. Unfolding the band structure of GaAsBi. J. Phys. Condens. Matter 2016, 29, 075001. [CrossRef]

41. Dargam, T.G.; Capaz, R.B.; Koiller, B. Disorder and size effects in the envelope-function approximation. Phys. Rev. B 1997, 56, 9625-9629. [CrossRef]

42. Boykin, T.B.; Klimeck, G. Practical application of zone-folding concepts in tight-binding calculations. Phys. Rev. B 2005, 71, 115215. [CrossRef]

43. Popescu, V.; Zunger, A. Effective band structure of random alloys. Phys. Rev. Lett. 2010, 104, 236403. [CrossRef]

44. Chen, M.; Weinert, M. Layer k-projection and unfolding electronic bands at interfaces. Phys. Rev. B 2018, 98, 245421. [CrossRef]

45. Lee, C.C.; Yamada-Takamura, Y.; Ozaki, T. Unfolding method for first-principles LCAO electronic structure calculations. J. Phys. Condens. Matter 2013, 25, 345501. [CrossRef] [PubMed]

46. Ku, W.; Berlijn, T.; Lee, C.C. Unfolding first-principles band structures. Phys. Rev. Lett. 2010, 104, 216401. [CrossRef]

47. Allen, P.B.; Berlijn, T.; Casavant, D.A.; Soler, J.M. Recovering hidden Bloch character: Unfolding electrons, phonons, and slabs. Phys. Rev. B 2013, 87, 085322. [CrossRef]

48. Rubel, O.; Bokhanchuk, A.; Ahmed, S.J.; Assmann, E. Unfolding the band structure of disordered solids: From bound states to high-mobility Kane fermions. Phys. Rev. B 2014, 90, 115202. [CrossRef]

49. Medeiros, P.V.C.; Stafström, S.; Björk, J. Effects of extrinsic and intrinsic perturbations on the electronic structure of graphene: Retaining an effective primitive cell band structure by band unfolding. Phys. Rev. B 2014, 89, 041407. [CrossRef]

50. Medeiros, P.V.C.; Tsirkin, S.S.; Stafström, S.; Björk, J. Unfolding spinor wave functions and expectation values of general operators: Introducing the unfolding-density operator. Phys. Rev. B 2015, 91, 041116. [CrossRef]

51. Huang, H.; Zheng, F.; Zhang, P.; Wu, J.; Gu, B.L.; Duan, W. A general group theoretical method to unfold band structures and its application. New J. Phys. 2014, 16, 033034. [CrossRef]

52. Kosugi, T.; Nishi, H.; Kato, Y.; Matsushita, Y.i. Periodicity-free unfolding method of electronic energy spectra. J. Phys. Soc. Jpn. 2017, 86, 124717. [CrossRef]

53. Mayo, S.G.; Yndurain, F.; Soler, J.M. Band unfolding made simple. J. Phys. Condens. Matter 2020, 32, 205902. [CrossRef]

54. Dombrowski, D.; Jolie, W.; Petrović, M.; Runte, S.; Craes, F.; Klinkhammer, J.; Kralj, M.; Lazić, P.; Sela, E.; Busse, C. Energy-dependent chirality effects in quasifree-standing graphene. Phys. Rev. Lett. 2017, 118, 116401. [CrossRef] [PubMed] 
55. Warmuth, J.; Bruix, A.; Michiardi, M.; Hänke, T.; Bianchi, M.; Wiebe, J.; Wiesendanger, R.; Hammer, B.; Hofmann, P.; Khajetoorians, A.A. Band-gap engineering by Bi intercalation of graphene on $\operatorname{Ir}(111)$. Phys. Rev. B 2016, 93, 165437. [CrossRef]

56. Abdollahi, A.; Golzan, M.M.; Aghayar, K. Electronic properties of GaxIn1-xP ternary alloy from first-principles. Comput. Mater. Sci. 2016, 120, 70-76. [CrossRef]

57. Molina-Sánchez, A.; Sangalli, D.; Hummer, K.; Marini, A.; Wirtz, L. Effect of spin-orbit interaction on the optical spectra of single-layer, double-layer, and bulk MoS 2 . Phys. Rev. B 2013, 88, 045412. [CrossRef]

58. Sipe, J.E.; Shkrebtii, A.I. Second-order optical response in semiconductors. Phys. Rev. B 2000, 61, 5337-5352. [CrossRef]

59. Salazar, C.; Cheng, J.L.; Sipe, J.E. Coherent control of current injection in zigzag graphene nanoribbons. Phys. Rev. B 2016, 93, 075442. [CrossRef]

60. Lee, M.H.; Yang, C.H.; Jan, J.H. Band-resolved analysis of nonlinear optical properties of crystalline and molecular materials. Phys. Rev. B 2004, 70, 235110. [CrossRef]

61. Nastos, F.; Olejnik, B.; Schwarz, K.; Sipe, J.E. Scissors implementation within length-gauge formulations of the frequency-dependent nonlinear optical response of semiconductors. Phys. Rev. B 2005, 72, 045223. [CrossRef]

62. Stahrenberg, K.; Herrmann, T.; Wilmers, K.; Esser, N.; Richter, W.; Lee, M.J.G. Optical properties of copper and silver in the energy range 2.5-9.0 eV. Phys. Rev. B 2001, 64, 115111. [CrossRef]

63. Reshak, A.H.; Chen, X.; Auluck, S.; Kamarudin, H. Linear and nonlinear optical susceptibilities and hyperpolarizability of borate LiNaB4O7 single crystals: Theory and experiment. J. Appl. Phys. 2012, 112, 053526. [CrossRef]

64. Okoye, C.M.I. Theoretical study of the electronic structure, chemical bonding and optical properties of KNbO3in the paraelectric cubic phase. J. Phys. Condens. Matter 2003, 15, 5945-5958. [CrossRef]

65. Leitsmann, R.; Schmidt, W.G.; Hahn, P.H.; Bechstedt, F. Second-harmonic polarizability including electron-hole attraction from band-structure theory. Phys. Rev. B 2005, 71, 195209. [CrossRef]

66. Gonze, X.; Jollet, F.; Araujo, F.A.; Adams, D.; Amadon, B.; Applencourt, T.; Audouze, C.; Beuken, J.M.; Bieder, J.; Bokhanchuk, A.; et al. Recent developments in the ABINIT software package. Comput. Phys. Commun. 2016, 205, 106-131. [CrossRef]

67. Gonze, X.; Amadon, B.; Anglade, P.M.; Beuken, J.M.; Bottin, F.; Boulanger, P.; Bruneval, F.; Caliste, D.; Caracas, R.; Côté, M.; et al. ABINIT: First-principles approach to material and nanosystem properties. Comput. Phys. Commun. 2009, 180, 2582-2615. [CrossRef]

68. Hamann, D.R. Optimized norm-conserving Vanderbilt pseudopotentials. Phys. Rev. B 2013, 88, 085117. [CrossRef]

69. Perdew, J.P.; Burke, K.; Ernzerhof, M. Generalized gradient approximation made simple. Phys. Rev. Lett. 1996, 77, 3865-3868. [CrossRef]

70. Monkhorst, H.J.; Pack, J.D. Special points for Brillouin-zone integrations. Phys. Rev. B 1976, 13, 5188-5192. [CrossRef]

71. Persson, K. Materials Data on KCl (SG:225) by Materials Project, 2014; Lawrence Berkeley National Lab: Berkeley, CA, USA, 2014. [CrossRef]

72. Roessler, D.M.; Walker, W.C. Electronic Spectra of Crystalline NaCl and KCl. Phys. Rev. 1968, 166, 599-606. [CrossRef]

73. Phillips, J.C. Ultraviolet Absorption of Insulators. III. fcc Alkali Halides. Phys. Rev. 1964, 136, A1705-A1713. [CrossRef]

74. Jones, R.O.; Gunnarsson, O. The density functional formalism, its applications and prospects. Rev. Mod. Phys. 1989, 61, 689-746. [CrossRef]

75. Boykin, T.B.; Kharche, N.; Klimeck, G.; Korkusinski, M. Approximate bandstructures of semiconductors alloys from tight-binding supercell calculations. J. Phys. Condens. Matter 2007, 19, 036203. [CrossRef]

76. Dai, Z.H.; Xie, Y.P.; Qian, Y.C.; Hu, L.J.; Li, X.D.; Ma, H.T. Effects of P, As, and Sb heavy doping on band gap narrowing of germanium as light emmiting materials. arXiv 2017, arXiv:1706.04050.

77. Deretzis, I.; Calogero, G.; Angilella, G.G.N.; Magna, A.L. Role of basis set on the unfolding of supercell band structure: From tight-binding to density functional thoery EPL. Europhys. Lett. 2014, 107, 27006. [CrossRef]

78. Onida, G.; Reining, L.; Rubio, A. Electronic excitations: Density-functional versus many-body Greeen's function approaches. Rev. Mod. Phys. 2002, 74, 601-659. [CrossRef] 
79. Hybertsen, M.S.; Louie, S.G. Electron correlation in semiconductors and insulators: Band gaps and quasiparticule energies. Phys. Rev. B 1986, 34, 5390-5413. [CrossRef]

80. Fox, M. Optical Properties of Solids. In Oxford Master Series in Condensed Matter Physics; Oxford University Press: New York, NY, USA, 2001; p. 288.

(C) 2020 by the authors. Licensee MDPI, Basel, Switzerland. This article is an open access article distributed under the terms and conditions of the Creative Commons Attribution (CC BY) license (http://creativecommons.org/licenses/by/4.0/). 